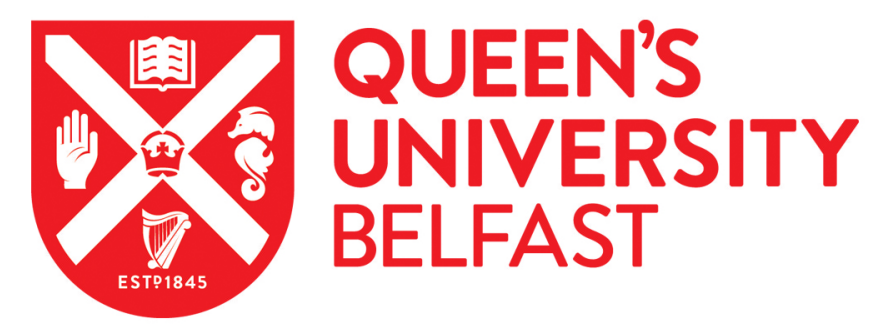

\title{
Use of SELDI MS to discover and identify potential biomarkers of toxicity in InnoMed PredTox: A multi-site, multi-compound study
}

Collins, B. C., Sposny, A., McCarthy, D., Brandenburg, A., Woodbury, R., Pennington, S. R., Gautier, J-C., Hewitt, P., \& Gallagher, W. M. (2010). Use of SELDI MS to discover and identify potential biomarkers of toxicity in InnoMed PredTox: A multi-site, multi-compound study. Proteomics, 10(8), 1592-1608.

https://doi.org/10.1002/pmic.200900608

Published in:

Proteomics

Document Version:

Peer reviewed version

Queen's University Belfast - Research Portal:

Link to publication record in Queen's University Belfast Research Portal

Publisher rights

Copyright @ 2010 WILEY-VCH Verlag GmbH \& Co. KGaA, Weinheim. This work is made available online in accordance with the publisher's policies. Please refer to any applicable terms of use of the publisher.

\section{General rights}

Copyright for the publications made accessible via the Queen's University Belfast Research Portal is retained by the author(s) and / or other copyright owners and it is a condition of accessing these publications that users recognise and abide by the legal requirements associated with these rights.

Take down policy

The Research Portal is Queen's institutional repository that provides access to Queen's research output. Every effort has been made to ensure that content in the Research Portal does not infringe any person's rights, or applicable UK laws. If you discover content in the Research Portal that you believe breaches copyright or violates any law, please contact openaccess@qub.ac.uk. 


\section{Research Article}

Use of SELDI Mass Spectrometry to Discover and Identify Potential Biomarkers of Toxicity in InnoMed PredTox: a Multi-Site, Multi-Compound Study.

\section{Authors}

Ben C. Collins ${ }^{1}$, Alexandra Sposny ${ }^{2}$, Diane McCarthy ${ }^{3}$, Arnd Brandenburg ${ }^{4}$, Ronald Woodbury ${ }^{3}$, Stephen R. Pennington ${ }^{5}$, Jean-Charles Gautier ${ }^{6}$, Phillip Hewitt ${ }^{2}$, and William M. Gallagher ${ }^{1}$.

${ }^{1}$ UCD School of Biomolecular and Biomedical Science and ${ }^{5}$ UCD School of Medicine and Medical Science, UCD Conway Institute, University College Dublin, Ireland; ${ }^{2}$ nstitute for Toxicology, MerckSerono, Darmstadt, Germany; ${ }^{3}$ Bio-Rad Laboratories, Malvern, PA, USA; ${ }^{4}$ Genedata AG, Basel, Switzerland; ${ }^{6}$ Sanofi-Aventis, Vitry sur Seine, France.

\section{Corresponding author}

William M. Gallagher

UCD School of Biomolecular and Biomedical Science, UCD Conway Institute, Belfield, Dublin 4, Ireland.

Email: william.gallagher@ucd.ie, Fax: +353 12837211 


\section{Abbreviations}

2D-DIGE, 2-dimensional difference in gel electrophoresis; 2D-PAGE, 2-dimensional polyacylamide gel electrophoresis; ACN, acetonitrile; CV, coefficient of variation; EDTA, ethylenediaminetetraacetic acid ; EFPIA, European Federation of Pharmaceutical Industries and Associations; ELISA, enzymelinked immunosorbent assay; EMEA, European Medicines Agency; EU, European Union; FDA, United States Food and Drug Administration; IPA, isopropyl alcohol; LC-MS, liquid chromatography mass spectrometry; MeOH, methanol; MES, 2-(N-morpholino)ethanesulfonic acid; MALDI-TOF/TOF, matrix assisted laser desorption/ionisation tandem time of flight mass spectrometry; nLC-MS/MS, nanoflow liquid chromatography tandem mass spectrometry; NMR, nuclear magnetic resonance spectroscopy; OGP, octyl $\beta$-D-glucopyranoside; SDS-PAGE, sodium dodecyl sulfate polyacrylamide gel electrophoresis; SELDI-TOF-MS (also referred to as SELDI), surface enhanced laser desorption/ionisation - time of flight - mass spectrometry;

\section{Keywords}

Toxicoproteomics, pre-clinical safety, SELDI-TOF-MS, systems toxicology

\section{Abstract}

A serious bottleneck in the drug development pipeline is the inability of current preclinical toxicology evaluation methods to predict early on, and with good accuracy, that a drug candidate will have to be removed from development due to toxicology/safety issues. The InnoMed PredTox consortium attempted to address this issue by assessing the value of using molecular profiling techniques (proteomics, transcriptomics, metabonomics), in combination with conventional toxicology measurements, on decision making earlier in preclinical safety evaluation. Here, we report on the 
SELDI-TOF-MS proteomics component of the InnoMed PredTox project. In this large scale, multi-site, multi-compound study, tissue and plasma samples from 14 day in vivo rat experiments conducted for 16 hepato- and nephro-toxicants with known toxicology endpoints (including 14 proprietary compounds and 2 reference compounds) were analysed by SELDI-TOF-MS. We have identified 7 plasma proteins and 4 liver proteins which were shown to be modulated by treatment, and correlated with histopathological evaluations and can be considered potential biomarker candidates for the given toxicology endpoints. In addition, we report on the intra- and inter-site variations observed based on measurements from a reference sample, and steps that can be taken to minimise this variation.

\section{Introduction}

Failure of experimental drug candidates due to toxicity issues remains a significant problem for pharmaceutical companies engaged in drug development activities [1]. There is a pressing need for improved pre-clinical toxicology in the early evaluation of novel drug candidates to reduce the rate of attrition in later stages of development (or indeed failures at the post-marketing stage) due to toxicity/safety issues [2]. This need is underlined by the increasing cost of bringing a new therapeutic molecule to market, estimated in 2003 to be approximately US $\$ 800$ million, although current thinking would suggest this has risen beyond US \$1 billion [3]. The desire to supplement the gold standard toxicology evaluation methods, such as histopathology and clinical chemistry, with more informative and predictive techniques has led to the engagement by drug developers of advanced molecular profiling technologies (transcriptomics, proteomics, metabolomics, etc.) to provide new insights into the mechanisms underlying toxicologic pathologies. The adoption of these technologies, combined with advanced computational methods, has spawned the novel field of 'systems toxicology' $[4,5]$. The primary aims of this new discipline can be summarised as, (i) to develop a 
more refined understanding of molecular mechanisms related to specific toxicologies, and, (ii) to elucidate molecular signatures associated with the prediction of those toxicological events (i.e. biomarkers or panels of biomarkers). This effort was initially led by transcriptomics experiments [6, 7]; however, more recently, proteomic (and indeed metabolomic) screens are increasingly employed. The sub-discipline of toxicoproteomics [8-10] is emerging as an integral component of the systems toxicology approach [11].

A growing trend in the pre-clinical toxicology arena is the emergence of consortia focused on applying combined 'omics approaches [12]. This phenomenon is likely due to the realisation that a single company or institution alone does not have the resources to profile a sufficient number of toxicologically well-defined compounds with which to build a reference knowledgebase. One such consortium is InnoMed PredTox ( $\underline{w w w . i n n o m e d-p r e d t o x . c o m}$ ), a collaborative project partly funded by the EU, and co-ordinated by EFPIA (European Federation of Pharmaceutical Industries and Associations), involving a consortium of 15 industrial (13 large pharma, 1 technology provider and 1 SME) and 3 academic partners. The stated aims of the project were to (i) enable more informed decision making earlier in preclinical safety evaluation by combining results from 'omics technologies together with conventional toxicology methods, (ii) promote the development of scientists within the novel field of systems toxicology, and (iii) to review this process with relevant regulatory authorities. The consortium has recently completed a 40 month project involving the evaluation of 14 compounds removed from development due to toxicity issues (these compounds were sponsored by the PredTox pharma partners), and 2 reference compounds with known toxicology, using a battery of molecular profiling methods. In vivo rat studies were carried out, and tissue and peripheral fluid samples were subjected to interrogation by transcriptomics (Affymetrix microarrays), proteomics (SELDI-TOF-MS, 2D-PAGE, 2D-DIGE) and metabolomics (LC-MS, NMR), along with measurement of standard toxicology endpoints [13]. This report deals specifically with 
the results of the SELDI proteomics component of the PredTox project (further manuscripts, which will describe other aspects of this collaborative project, are in preparation).

SELDI-TOF-MS utilises chemically derivatised arrays to chromatographically bind a subset of proteins from a biological matrix of interest, and time-of-flight mass spectrometry to profile the bound peptides/proteins in a semi-quantitative manner [14]. SELDI has been adopted as a proteomic profiling method, in particular, by researchers conducting clinical/pre-clinical studies in which large numbers of samples are analysed and more standard proteomic approaches (such as 2-dimensional electrophoresis or LC-MS/MS) are not suitable due to their lower throughput. It is worth noting that although SELDI can provide higher throughput, and so greater statistical rigour, it has been criticised with regard to the power of the technique in terms of its ability to profile anything other than the medium to high abundance proteins in a given sample. Put more simply, there is a trade-off between throughput and depth of proteomic profiling. The potential of SELDI to be used robustly in a multicenter fashion was previously demonstrated by the Human Plasma Proteome Project [15] and several groups have reported on the use of SELDI in a pre-clinical toxicology context [16-19]. The aim of this study was to profile the target organ of toxicity (liver or kidney) and plasma of approximately 480 rodents treated with previously characterised toxic compounds, at various dosages and time points, in order to elucidate candidate protein biomarkers associated with particular toxicologic pathologies that could be useful in predicting that pathology in future pre-clinical studies.

\section{Materials and methods}

\subsection{Compound selection and in vivo studies}

Fourteen compounds which had been previously removed from development due to hepato- or nephrotoxicity issues in late pre-clinical, or early clinical development, were donated by the InnoMed PredTox partners for use in this project. Two previously described toxicants, troglitazone 
(hepatotoxicant) in study FP010SG and gentamicin (nephrotoxicant) in study FP009SF, were also included as reference compounds. A total of 16 in vivo studies were performed according to a standardised protocol at a variety of InnoMed PredTox partner sites (see Supplementary Table 1 for the study and compound list). Animal experimentation plans underwent an ethical review within the project and were carried out according to the local regulations and permissions of each participant company and in accordance with the guidelines of the European Council on Experimental Animal Care. Male Wistar rats aged 8-10 weeks and weighing 170g-200g at the study commencement were administered a daily treatment of the selected compound at vehicle, low (sub-toxic), or high (toxic) dose for 1,3 , or 14 days. Each of the 9 study groups contained 5 animals (45 animals per compound study - see Supplementary Table 2 for the detailed treatment schedule). Necropsy was performed 24 hours after the final dose $(2,4$, or 15 days). Standard toxicology analyses including observations/body weight, serum clinical chemistry, haematology, urinalysis, organ weight, and histopathology were carried out, and the liver, kidneys, urine and blood were collected for subsequent transcriptomics, metabolomics, and proteomics analysis. For the purpose of SELDI proteomic studies, plasma was prepared from approximately $1.5 \mathrm{ml}$ whole blood by centrifugation in pre-cooled EDTA coated tubes at $2000 \times \mathrm{g}, 4^{\circ} \mathrm{C}$, for $15 \mathrm{~min}$. Approximately $0.75 \mathrm{ml}$ of plasma was recovered (supernatant) and stored at $-80^{\circ} \mathrm{C}$. The liver and kidneys were dissected and snap frozen. The left lateral lobe of the liver and the upper half of the right kidney were used for SELDI proteomics studies.

\subsection{Site-to-site harmonisation}

The SELDI proteomics studies were performed using a standardised protocol in the laboratories of 3 of the InnoMed PredTox partners: University College Dublin, Ireland; Merck-Serono, Germany; and Bio-Rad Laboratories, Denmark and USA. All SELDI arrays, binding buffers and sinapinic acid matrix 
used throughout the profiling study were purchased from Bio-Rad Laboratories and were from the same or consecutive lots. ProteoExtract albumin/lgG depletion kits from the same lot were purchased from Calbiochem (Germany). Buffers for tissue lysis were prepared in Merck-Serono and distributed to the other laboratories. Scientists from all 3 institutions undertook visits to the other laboratories to compare and harmonise analytical techniques. Finally, a reference sample consisting of rat EDTA plasma was purchased from Taconic (Denmark) and distributed to each laboratory to serve as a quality standard for site-to-site comparisons.

\subsection{Protein extraction and depletion of abundant species from plasma}

In order to increase the number of features detectable by SELDI-TOF-MS, plasma was depleted of the two most abundant proteins, namely albumin and IgG, using the ProteoExtract ${ }^{\circledast}$ Albumin/lgG Removal Kit (Calbiochem) according to the manufacturer's instructions. Briefly, $35 \mu$ l of plasma were diluted to $350 \mu \mathrm{l}$ with binding buffer containing $1.8 \mu \mathrm{l}$ protease inhibitors (Merck PI set III). After the column was equilibrated the diluted sample was loaded onto the column. The flow-through and $2 \mathrm{x}$ $600 \mu$ l binding buffer washes were collected in a fresh tube (depleted fraction). Elution of the bound proteins was attempted with $1000 \mu \mathrm{l} 2.5 \mathrm{M} \mathrm{NaCl}, 25 \mathrm{mM}$ sodium phosphate, pH 8.0 (albumin fraction) and $1000 \mu \mathrm{l} 250 \mathrm{mM}$ citric acid (IgG fraction). The protein from the depleted fraction of plasma was concentrated and desalted by acetone precipitation for 3 hours at $-20^{\circ} \mathrm{C}$ in 4.5 volumes of acetone which had been pre-cooled to $-20^{\circ} \mathrm{C}$. The precipitated protein was pelleted by centrifugation ( $5000 \times \mathrm{g}, 10 \mathrm{~min}, 4^{\circ} \mathrm{C}$ ), the acetone supernatant was aspirated, and the pellet was resolubilised in $200 \mu \mathrm{l}$ U9 buffer (9M urea, 2\% CHAPS, $50 \mathrm{mM}$ Tris-HCl pH 9.0). The protein concentration was estimated using the method described by Bradford [20] with Bioquant reagent (Merck Biosciences, Germany). The depleted plasma sample was aliquoted and stored at $-80^{\circ} \mathrm{C}$. 


\subsection{Protein extraction from tissue}

Protein extraction from tissues was achieved using a protocol adapted from a method previously optimised at Merck-Serono [16, 21]. The frozen liver left lateral lobe $(100 \pm 50 \mathrm{mg})$ was ground with a mortar and pestle under liquid nitrogen. Powdered tissue was transferred into a tube containing $125 \mu$ l lysis buffer I (10 mM Tris, pH 7.5, 1 mM EDTA, 0.2 M saccharose, Benzonase and inhibitor cocktail) and completely suspended. Afterward, $875 \mu$ l lysis buffer II (7 M urea, 2 M thiourea, 4\% CHAPS, $40 \mathrm{mM}$ DTT, $20 \mathrm{mM}$ spermine) was added and pipetted to aid suspension. The protein extracts were mixed at room temperature on a rotary shaker at $500 \mathrm{rpm}$ to ensure as complete as possible cell lysis, and solubilisation of protein. To separate membranous components and other insoluble debris, samples were ultra-centrifuged for $30 \mathrm{~min}$ at $10^{\circ} \mathrm{C}$ and $74,000 \times$ g. Protein concentrations of the supernatants were determined by Bradford assay as above and the samples were aliquoted for storage at $-80^{\circ} \mathrm{C}$. Protein extraction from kidney samples was as described for liver with the exceptions that the entire upper right half of the kidney was used and the buffer volumes were doubled.

\subsection{SELDI-TOF-MS profiling}

The CM10 (weak cation exchange) and Q10 (strong anion exchange) surfaces were chosen due to the large number of $\mathrm{m} / \mathrm{z}$ features observed in previous in-house studies and optimisation experiments (data not shown). Low stringency binding buffers were used for both surfaces, again, to maximise the number of $\mathrm{m} / \mathrm{z}$ features observed. The binding buffer for the $\mathrm{CM} 10$ surface was 100 $\mathrm{mM}$ sodium acetate $\mathrm{pH} 4.0$ and the binding buffer for the $\mathrm{Q} 10$ surface was $50 \mathrm{mM}$ Tris- $\mathrm{HCl} \mathrm{pH} 9.0$. The samples were applied to the arrays using 4 technical replicates in a randomised fashion (see Supplementary Figure 1) and the reference sample was included on one spot of every array for quality control purposes. Spots for laser optimisation and buffer only controls were also included. 
The arrays were prepared essentially according to the manufacturer's instructions. Briefly, the arrays were equilibrated with mixing using $150 \mu$ of the appropriate binding buffer for $2 \times 5$ minutes. $30 \mu \mathrm{g}$ of protein extracted from tissue, or $20 \mu \mathrm{g}$ of protein from depleted plasma, were diluted to $150 \mu \mathrm{l}$ with binding buffer, applied to the arrays and incubated for $45 \mathrm{~min}$ at room temperature with mixing (DPC Micromix). The buffer was discarded and the arrays were washed 3 times with $150 \mu$ l binding

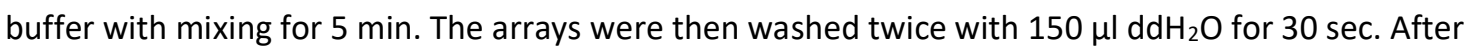
the wash was discarded the arrays were dried with gentle heating $\left(40^{\circ} \mathrm{C}\right)$ for $15 \mathrm{~min}$. A saturated solution of sinapinic acid in $0.5 \%(\mathrm{v} / \mathrm{v})$ trifluroacetic acid, $50 \%(\mathrm{v} / \mathrm{v})$ acetonitrile was prepared and 1 $\mu \mathrm{l}$ was applied to each spot, allowed to dry, and $1 \mu \mathrm{l}$ was again applied and allowed to dry. All spectra were acquired using a PCS-4000 SELDI-TOF mass spectrometer (Bio-Rad Laboratories) using two protocols which were optimised for low and high mass ranges (see Supplementary Table 3). As the laser and detector performance changes over time, optimal settings were determined empirically for each site and instrument. A set of nominal $\mathrm{m} / \mathrm{z}$ features and corresponding intensities in the reference sample were designated and used to assist with laser intensity optimisation at each site (see Supplementary Table 4). All sample spectra were mass calibrated with reference to spectra acquired from a standard protein mixture (Protein MW Standards, Bio-Rad Laboratories) collected on the same day as the sample data. In total, 17,920 SELDI spectra were acquired including 7,680 spectra derived from depleted plasma samples, 5,760 spectra derived from liver samples, 1,920 spectra derived from kidney samples, and 2,560 spectra derived from the reference sample and buffer controls. All raw and processed spectra in .xml and .csv formats were exported and submitted for central storage in the PredTox relational database housed at Genedata AG (Basel, Switzerland).

\subsection{Data processing and statistical analyses}


The data processing and statistical analyses were performed in parallel using two separate software platforms and workflows. The first analysis was carried out at University College Dublin, MerckSerono, and Bio-Rad Laboratories using ProteinChip DataManager version 3.06 (Bio-Rad Laboratories) and the second was carried out at Genedata AG using the Genedata Expressionist ${ }^{\circledR}$ platform with the Refiner MS and Analyst modules.

\subsubsection{ProteinChip DataManager Analysis}

The raw data were pre-processed by baseline subtraction, filtering, noise measurement, total ion current normalisation, and alignment (see Supplementary Table 5 for details). The median and standard deviation of the internal normalisation coefficients was calculated for each subgroup (i.e. for the same compound study, sample material, array surface and mass range). As a quality control measure, spectra which had an internal normalisation coefficient greater than 2 times the median were removed (a large internal normalisation coefficient, indicating a small total ion current, can be caused by incomplete binding of the sample to the array due to an air bubble or other experimental error). Principal components analysis was used to visualise variance in the data. The peak detection tool was run (see Supplementary Table 6 for details) and an initial low stringency statistical analysis was performed. Peaks which yielded a $p$-value $<0.02$ and/or a receiver operating characteristic area under the curve of $>0.8$ in the treated animals versus the time-matched vehicle control were prioritised for further analysis. A manual inspection of the peak quality and peak labelling for the prioritised peaks was performed for every spectrum in the dataset and peaks considered to be spurious or of low quality were removed from the list. A second, more stringent, round of statistical analysis was then undertaken where two statistical cut-offs were employed (i) Mann-Whitney pvalue $<0.01$ and median fold change $>2$, (ii) Mann-Whitney $p$-value $<0.05$ and median fold change $>$ 1.5. The lower stringency cut-off was taken into account for conditions which did not yield peaks 
that met the higher stringency criteria and when considering peaks which were modulated across more than one compound study or where the same mass was modulated in plasma and tissue from the same compound study. This second, less stringent cut off was included to reflect the increased power due to a larger sample size when comparing across multiple studies, sample types (i.e. tissue or plasma), or time points (a formal power analysis was not carried out due to the complexity of comparing across multiple study groups, sample types, and time points).

\subsubsection{Genedata Expressionist ${ }^{\circledR}$ Refiner MS and Analyst}

Preprocessing of the raw data and statistical analyses were also carried out independently using Genedata Expressionist ${ }^{\circledR}$. The Refiner MS module of Genedata Expressionist ${ }^{\circledR}$ (version Pro 4.5, Genedata AG) was used to perform several processing steps for all studies and for each of the available sets of spectra (low/high energy, Q10/CM10, plasma/target organ) separately. Spectra were aligned in the $\mathrm{m} / \mathrm{z}$ dimension so that their respective $\mathrm{m} / \mathrm{z}$ values could be reliably compared. A baseline subtraction was performed to get spectra that have zero as their lowest intensity level. A median normalisation was carried out to rescale the intensity values of the raw spectra such that they become comparable. Peaks corresponding to $\mathrm{m} / \mathrm{z}$ intervals with a central mass value were detected using a curvature-based algorithm. A signal-to-noise measure was computed for each peak. Finally, peaks from several spectra were used to determine a common set of peaks subsequently used throughout all spectra. This step was crucial for creating a data matrix of peak intensities amenable to statistical analysis. Statistical analyses were performed using the Analyst module of Genedata Expressionist ${ }^{\circledast}$. Only peaks with a signal-to-noise ratio of at least 2.5 were considered. The four technical replicate spectra available for each sample were combined by taking the median intensity per peak. A median normalisation was applied to the combined spectra. To obtain an overview of the separation of the data according to time and dose, principal components were 
computed and visualised. The statistical analysis comprised 2-way ANOVA using time and dose as factors, t-tests and fold change analyses between time-matched groups of treated and control samples. Tables with $p$-values and fold changes were generated for all studies. These results were used to obtain the peaks with highest fold changes and most significant $p$-values and to cross-check the significance of peaks found using Bio-Rad's ProteinChip DataManager software. They also served as the starting point to find common peaks across different studies, as well as peaks present both in plasma and liver/kidney tissue.

\subsection{Purification of candidate biomarker proteins for identification}

Several separation techniques were employed for purification of target proteins from tissue extracts and depleted plasma samples. Samples containing high and low levels of the target protein were pooled to create a positive and negative pool and processed in parallel, allowing confirmation of differential expression at each step of the purification process. The presence and relative purity of the target protein was monitored at every stage by SELDI-TOF-MS. Samples from the same experimental group were pooled for the purpose of scale-up for identification. The pooled samples from the experimental groups in which a target protein was differentially modulated were fractionated in parallel to ensure that the target peak maintained a comparable fold change to add confidence to the mass assignment.

\subsubsection{Off gel electrophoresis (preparative isoelectric focusing)}

$2-10 \mathrm{mg}$ of protein sample from depleted plasma or tissue extract was diluted into $3.6 \mathrm{ml}$ of the offgel electrophoresis buffer $(6.88 \mathrm{M}$ urea, $1.84 \mathrm{M}$ thiourea, $1.12 \% \mathrm{w} / \mathrm{v}$ dithiothreitol, $9.6 \% \mathrm{v} / \mathrm{v}$ Glycerol, $0.96 \% \mathrm{v} / \mathrm{v}$ ampholytes $\mathrm{pH}$ range 3-10) and applied to $\mathrm{pH}$ 3-10 non-linear immobilised $\mathrm{pH}$ 
gradient gel strips (GE Healthcare) in 24 partitions. In some cases, de-salting was required prior to off-gel electrophoresis and this was achieved with a buffer exchange using Amicon Ultra-4 $3 \mathrm{kDa}$ molecular weight cut-off filters (Millipore). The protein samples were focused for 100,000 volt hours (current $=50 \mu \mathrm{A}$, max. voltage $=6000 \mathrm{~V}$ ) using the Agilent 3100 OFFGEL fractionator and recovered in 24 fractions of approximately $150 \mu \mathrm{l}$.

\subsubsection{Strong anion exchange chromatography}

$\mathrm{Q}$ HyperD $^{\circ} \mathrm{F}$ resin (Pall Corporation) was equilibrated with $50 \mathrm{mM}$ Tris- $\mathrm{HCl}$, pH 9. Plasma or liver lysates was diluted in $50 \mathrm{mM}$ Tris, $\mathrm{pH} 9.0$ and bound to Q HyperD F resin, either in batch format or in a spin column. After incubating on an end-over end mixer at room temperature for a minimum of 30 min, the flow through was collected and the resin washed with $50 \mathrm{mM}$ Tris, $\mathrm{pH} 9,0.1 \%$ OGP. Bound proteins were eluted successively with the following buffers: $50 \mathrm{mM} \mathrm{HEPES,} \mathrm{pH} \mathrm{7,} 0.1 \%(\mathrm{w} / \mathrm{v})$ OGP;

$100 \mathrm{mM}$ sodium acetate, $\mathrm{pH}$ 5, $0.1 \%$ (w/v) OGP; $100 \mathrm{mM}$ sodium acetate, $\mathrm{pH}$ 4, 0.1\% (w/v) OGP; 50 mM sodium citrate, $\mathrm{pH} 3,0.1 \%(\mathrm{w} / \mathrm{v})$ OGP and 33\% (v/v) IPA, $17 \%(\mathrm{v} / \mathrm{v}) \mathrm{ACN}, 0.1 \%(\mathrm{v} / \mathrm{v})$ TFA. An aliquot of each fraction was diluted 10-fold and profiled on CM10 and/or Q10 ProteinChip arrays to assay for the proteins of interest.

\subsubsection{Reversed phase chromatography}

PLRP-S beads (Polymer Laboratories) were equilibrated with 5\% ACN/0.5\% TFA. An appropriate volume of each sample was adjusted to a final concentration of $5 \% \mathrm{ACN} / 0.5 \%$ TFA and mixed for a minimum of $30 \mathrm{~min}$ with the PLRP-S beads at room temperature. The tube was centrifuged and the supernatant collected. Bound proteins were eluted successively with 10\%, 15\%, 20\%, 25\%, 30\%, $35 \%, 40 \%, 50 \%, 60 \%$ and/or $70 \%$ ACN in $0.1 \%$ TFA (formic acid was also used as modifier). A $1-2 \mu \mathrm{L}$ 
aliquot of each eluate was profiled on an NP20 array to evaluate the total protein content. The fraction(s) containing the target protein were then concentrated by centrifugal evaporation.

\subsubsection{One-dimensional electrophoresis}

Once the protein of interest was sufficiently enriched, fractions were re-suspended in lithium or sodium dodecyl sulphate sample buffer (Novex) and subjected to 1-dimensional electrophoresis. Gel compositions were optimised for different molecular weight targets and included $10 \%, 12 \%$, and 15 $\%$ Tris- $\mathrm{HCl}$ gels for larger molecular weight targets and $16.5 \%$ Tris-Tricine gels for targets under 10 kDa. Proteins were visualised using a modified colloidal Coomassie blue stain [22] or a Colloidal Blue Staining kit (Invitrogen). Peptide gels were stained using Instant Blue (Novexin).

\subsubsection{Analytical extraction of protein from polyacrylamide gels}

Selected protein bands were excised from the polyacrylamide gel and extracted to confirm the $\mathrm{m} / \mathrm{z}$ of the protein prior to digestion. One quarter of the gel pieces were washed with $200 \mu$ l of $50 \%$ methanol/10\% acetic acid for $30 \mathrm{~min}$, dehydrated with $100 \mu \mathrm{L} \mathrm{ACN}$ for $15 \mathrm{~min}$, and extracted with 70 $\mu \mathrm{L} 50 \%$ formic acid/25\% ACN/15\% IPA by incubating for a minimum of $2 \mathrm{hr}$ at room temperature with vigorous shaking. A 2-3 $\mu \mathrm{L}$ aliquot of the extract was profiled on an NP20 ProteinChip array.

\subsection{Identification of candidate biomarkers by tandem mass spectrometry}

Proteins were identified by MALDI or ESI tandem mass spectrometry after digestion of proteins with trypsin. Biomarker candidates under 4 kDa were sequenced directly by MALDI-TOF/TOF mass spectrometry. 


\subsubsection{Protein digestion by trypsin}

The protein eluted from SDS-PAGE gel plugs was digested with sequencing grade modified porcine trypsin (Promega) in-solution and/or in-gel digestion of gel plugs extracted from the same band. The gel plugs were destained with $100 \mathrm{mM}$ ammonium bicarbonate, $50 \%(\mathrm{v} / \mathrm{v}$ ) ACN (or $400 \mu \mathrm{L}$ of $50 \%$ acetonitrile/100 mM ammonium bicarbonate $\mathrm{pH} 8$ ) with rotary mixing for $3 \times 30 \mathrm{~min}$. The samples (in gel and in solution) were optionally subjected to reduction ( $5 \mathrm{mM}$ dithiothreitol, $50 \mathrm{mM}$ ammonium bicarbonate, $30 \mathrm{~min}$, room temperature) and alkylation ( $10 \mathrm{mM}$ iodoacetamide, $50 \mathrm{mM}$ ammonium bicarbonate, $30 \mathrm{~min}$, room temperature, dark) to eliminate disulphide bridging at cysteine residues. The gel plugs were dehydrated with $50 \mu \mathrm{l} 100 \%(\mathrm{v} / \mathrm{v}) \mathrm{ACN}$ and dried with centrifugal evaporation. 5-10 ng/ $\mu \mathrm{L}$ Trypsin in $20 \mu \mathrm{l}$ of $50 \mathrm{mM}$ ammonium bicarbonate was added to the samples (in-gel and in solution) and incubated overnight at $37^{\circ} \mathrm{C}$. The digested samples were removed to fresh tubes and dried by centrifugal evaporation.

\subsubsection{Tandem mass spectrometry of tryptic digests}

Tryptic digests were analysed by nLC-MS/MS or MALDI-TOF/TOF mass spectrometry. For nLCMS/MS, the digests were resuspended in $0.1 \%(\mathrm{v} / \mathrm{v})$ formic acid and introduced to an LTQ linear ion trap mass spectrometer interfaced via electrospray to a Surveyor nano LC system (Thermo Scientific) using a 30 min gradient elution from $3 \%(v / v)$ ACN, $0.1 \%(v / v)$ formic acid to $40 \%$ ACN $(v / v), 0.1 \%$ (v/v) formic acid.

Single MS and MS/MS MALDI spectra were acquired on a 4800 MALDI TOF-TOF mass spectrometer (Applied Biosystems). Equal volumes of the tryptic digest and saturated CHCA were mixed and spotted on a MALDI target. Selected digests were also bound to $\mu \mathrm{C} 18$ Zip-Tips (Millipore), eluted in 
$50 \%$ ACN/0.1\% TFA, mixed with an equal volume of CHCA and spotted onto the MALDI target.

Spectra were collected from 600 to 4,000 Da in reflectron mode. After reviewing the spectra, specific ions were selected for MS/MS analysis.

MS/MS spectra were searched using Mascot (v 2.1 - Matrix Sciences) or Protein Pilot (v2.0 - Applied Biosystems) against the International Protein Index for rat (v 3.50) appended with common laboratory protein contaminants (containing 40,351 protein entries). In some cases NCBInr and/or SwissProt databases were also used in database searches with use of BioMart for accession mapping. Peak list generation was performed using Bioworks (v3.1 - Thermo Scientific) or GPS explorer (v3.6 - Applied Biosystems). Mass tolerances for database searching were 1.5 Da (LTQ) or 100 ppm (TOF-TOF) for precursor ions and 0.8 Da (LTQ) or 0.25 Da (TOF-TOF) for fragment ions. Other search parameters were as follows: carbamidomethylated cysteine as fixed modification (in cases where alkylation was employed) and oxidised methionine as a variable modification, and allowing 1 missed cleavages and semi-tryptic specificity. In general a Mascot ion score which indicated identity or significant homology at the $p<0.05$ significance level was required, and in no case was a Mascot ion score of less than 30 accepted. The resulting peptide and protein assignments from MALDI and ESI mass spectrometry were manually inspected for spectral match quality and sequence coverage in all cases. The predicted intact protein molecular weight (including predicted processing events) were cross checked with the experimentally determined molecular weights for increased confidence in protein identifications.

\subsubsection{Direct sequencing by MALDI-TOF/TOF}

Low molecular weight peptides $(<4 \mathrm{kDa})$ determined to be differentially regulated were partially purified using anion exchange spin columns and further enriched on a cation exchange (CM10) ProteinChip array. The native peptide was then analysed directly on a 4800 MALDI-TOF/TOF mass 
spectrometer (Applied Biosystems) modified to accommodate ProteinChip arrays. MS/MS spectra were searched using ProteinPilot software (Applied Biosystems) against the NCBI rat database using the 'no enzyme' option.

\subsection{Beta-2-microglobulin ELISA}

Beta-2-microglobulin was assayed, using a commercially available sandwich ELISA (PanaTest Rat Beta-2-microglobulin EIA kit, Gentaur), in liver protein extracts according to the manufacturer's instructions. Summary statistics were generated with SPSS (v 15.0).

\section{Results}

\subsection{Effect of depletion of abundant proteins from plasma}

The challenges posed by the dynamic range of protein concentration in plasma is well described [23]. In order to increase the effective number of proteins quantifiable by SELDI-TOF-MS profiling depletion of abundant proteins from plasma was undertaken. Significant, but not complete, depletion of albumin and IgG was observed in SELDI spectra from depleted plasma (see Figure 1). During method optimisation experiments, depletion was shown to increase the number of peaks detectable by between 50 and $82 \%$ (depending on peak detection parameters - see Supplementary Table 7). There was no significant increase in the mean $\% \mathrm{CV}$ of intensity of peaks across technical replicates between non-depleted (15.3\%) and depleted plasma (15.9\%). In addition, an attempt was made to elute the bound proteins for analysis; however, the elution of bound proteins was not intended with this commercial depletion system. The elution of bound protein fractions from the column using instructions provided by the manufacturer was found to have very high technical variation as measured by SELDI analysis in pilot experiments (data not shown). It is likely that the 
elution of these protein fractions was not complete and, as such, these samples were not included for analysis.

[Insert Figure 1 here]

\subsection{Intra- and inter-site reproducibility}

Variation within individual compound studies, and across multiple studies and sites of data collection, was measured with respect to a reference rat plasma sample distributed to each site and included on every array in the project. Figure 2 shows the variation within compound studies (as determined by the $\% \mathrm{CV}$ ) of the intensity of peaks in the SELDI spectra with respect to site of collection, array type, and sample type (i.e. tissue extract or depleted plasma). The global mean of the $\%$ coefficient of variation across all studies was determined to be $23.0 \%$. The spectra collected from the reference sample were also used to explore the effect of multisite data collection on the variation in the data set. Figure 3 shows a principal components analysis of the entire reference sample spectra set collected throughout the project, annotated by the site of data collection, and by the individual compound studies. It is clear that there is some degree of bias based on the site of data collection despite the significant efforts made to harmonise all of the data collection procedures. This is most easily explained in terms of differences between the instruments on which the spectra were collected. In particular, the absolute peak intensity for a given protein at a given concentration is related to the age and settings for the SELDI instrument's laser and detector. As such, the disparity between spectra from multiple sites is primarily manifested in peak intensity. Figure 4 shows representative spectra from all 3 sites which demonstrate that the same $\mathrm{m} / \mathrm{z}$ feature sets are measured at each site (albeit at differing intensities). An effort was made to compensate for this by adjusting the laser setting at each site with respect to the intensity of a nominated set of peaks in the reference sample; however, it is clear that this was not entirely successful. Indeed, 
significant site biases (most probably due largely to peak intensity differences) have been observed in a previous multi-site SELDI study [15]. Site bias effects in multi-institution molecular profiling studies are largely unavoidable and were also observed in the metabolomics and transcriptomics components of the PredTox project (manuscripts in preparation). The crucial point is that this does not preclude a cross-study analysis of the data if the appropriate methods are applied. In this study, each compound study was analysed at a single site, and the initial statistical analysis was performed within a single compound study. Peaks that exhibited significant differences based on statistics and fold-change were then cross-compared between studies. Since data was effectively normalised within each compound study, the site-to-site bias had no effect on the final outcome. It is also worth noting that even if the SELDI analysis were carried out at a single site (thereby eliminating site bias in the SELDI profiling data), there would still be very significant bias arising from the site effects from the in vivo dosing studies. This in vivo study site bias is not represented in the reference sample which was included only to measure analytical variation in the SELDI profiling.

\section{[Insert Figure 2, Figure 3, and Figure 4 here]}

\subsection{Modulated peaks in SELDI profiling data}

The Mann-Whitney $p$-value and the area under the ROC curve (AUC) were used to evaluate the ability of each significant peak to discriminate effects related to time and dose. The $p$-value refers to the probability that an observed difference between groups has occurred by chance alone. A $p$-value less than 0.05 indicates that the probability the observed difference is due to chance alone is less than $5 \%$. The ROC curve plots sensitivity versus 1 -specificity. The area under the curve is indicative of the utility of the marker to distinguish an experimental group, with an AUC of 0.5 indicating no separation between groups and a value of 1.0 representing $100 \%$ sensitivity and specificity. Those peaks that were significant in multiple conditions were noted. A statistical analysis was conducted 
for each treatment dose (vehicle vs low dose, vehicle vs high dose) at each time point and for each treatment group over time (day 4 vs day 15); however, most of the initial data analysis effort was focused on the day 15 high dose versus day 15 vehicle experimental groups. Peaks were deemed significant if they returned a $p$-value less than 0.05 and/or an AUC greater than 0.8. Peaks that showed significant differences at day 15 were further evaluated for peak quality, magnitude of change, and consistency of changes. Peaks found to be significantly modulated in these groups were then examined in the early time point and low dose groups to determine their potential time and dose dependency. The numbers of differentially regulated peaks in each compound study at two significance levels (fold change $<0.67$ or fold change $>1.5$ and Mann-Whitney $p$-value $<0.05$, and, fold change $<0.5$ or fold change $>2.0$ and Mann-Whitney $p$-value $<0.01$ ) are summarized in Figure 5. These calculations were based primarily on the results of the ProteinChip Manager analysis, and were cross-checked for consistency using the analysis based on Refiner MS and Analyst modules of Genedata Expressionist ${ }^{\oplus}$.

Figure 6 shows an example of a peak at 11,645 Da (subsequently identified as $\beta$-2-microglobulin) whose intensity was consistently increased on treatment across 3 compound studies (FP005ME, FP007SE, FP014SC) classified in the bile duct damage/necrosis histopathology group (the pathology subgroups are described in this section below). The fold change and Mann-Whitney $p$-values for treatment groups in the 3 studies are summarised in Table 1. The intensity of the vehicle control groups for the compound studies FP005ME and FP014SC are at a comparable level, as the SELDI data for both of these studies were collected at a single site (UCD). However, the SELDI data for the compound study FP007SE was collected at a different site (Merck-Serono), and this is reflected in the fact that the vehicle control (i.e. baseline) measurements of this peak are at significantly different intensities. This difference is explained by operator and instrument variation between sites (especially the laser intensity setting), and would make any cross-site comparison at the raw spectra level extremely problematic. This does not, however, preclude multi-site comparisons when 
considered at the level of fold change relative to the time matched vehicle control for a particular mass feature (which can be subsequently identified after a shortlist of candidates of interest is compiled). This was the approach taken in this study for cross-site comparison of SELDI data.

\section{[Insert Figure 5,Figure 6 and Table 1 here]}

For the purpose of cross compound analysis of the SELDI data (as well as data analysis in other 'omics disciplines in the PredTox project) the studies were divided into 3 groups based on the histopathological findings for each study - (i) liver hypertrophy, (ii) liver bile duct damage/necrosis, and (iii) nephrotoxicity. An article containing more specific details of the histopathological findings in the PredTox project has already been published [13] and, as such, will not be dealt with in detail here.

The process of identifying proteins of interest (i.e. differentially regulated proteins) from top-down proteomics methods like SELDI profiling studies is relatively laborious compared with bottom-up proteomics approaches and, therefore, appropriate criteria need to be developed to choose the candidates for identification that will provide the most insight into the biological question. In this case, we selected 3 categories for inclusion on the list of target peaks for identification: (i) greatest fold change plasma peak per compound study, (ii) peaks in plasma and tissue studies with the same $\mathrm{m} / \mathrm{z}$ (that could potentially represent the same protein), and (iii) peaks modulated in multiple compound studies (this was focused on the second toxicologic pathology grouping described above, liver bile duct damage/necrosis, and included peaks from both plasma and liver studies). This selection process yielded a shortlist of candidate peaks earmarked for identification. 


\subsection{Candidate biomarkers identified}

The workflow for identifying peaks determined to be differentially regulated in SELDI profiling studies involves purifying the protein of interest using standard separation steps such as anion exchange and reversed phase chromatography, preparative isoelectric focusing (off-gel electrophoresis), and/or 1 dimensional gel electrophoresis. The resulting fractions are assayed for the presence and purity of the target of interest by assaying each fraction for the protein of interest on ProteinChip arrays (including using the same array chemistry on which the candidate marker was originally discovered). In addition, the purification is carried out in parallel on samples (or pools) with high and low levels of the target protein (typically day 15, high dose and day 15, vehicle treated animal groups). The combination of a chromatographic assay and parallel purification permits unambiguous confirmation that the protein purified is the marker of interest. In cases where the fragment of the protein identified was not previously annotated, the pl and molecular weight were calculated from the amino acid sequence of the hypothesised fragment. The results of the identification efforts are summarized in Table 2 (plasma samples) and Table 3 (liver samples - no identification attempts were made for kidney samples). Figure 7 outlines the identification of the peak at $11,622 \mathrm{~m} / \mathrm{z}$ in plasma as $\beta$-2-microglobulin by preparative isoelectric focusing, reversed phase chromatography, 1-dimensional electrophoresis, analytical extraction, tryptic digestion, and nLC-MS/MS. Supplemental Figure 3 outlines the purification and identification of a low molecular weight peptide $(2,890 \mathrm{Da})$ by direct sequencing from a SELDI array by MALDI-TOF/TOF mass spectrometry.

[Insert Table 2, Table 3 and Figure 7 here]

\subsection{Beta-2-microglobulin ELISA}


Beta-2-microglobulin in protein extracts from liver was measured by an orthogonal method (commercially available ELISA) as a technical validation of the SELDI method. The trends between experimental groups as observed by SELDI and ELISA compare favourably. The trend of fold changes versus vehicle controls as measured by ELISA are essentially in good agreement with the SELDI data (see Table 4). The SELDI and ELISA results are well correlated at the individual animal level (Spearman's rank correlation coefficient=0.79).

[Insert Table 4 here]

\section{Discussion}

In this report, we have described in detail the steps taken to carry out a large scale, multi-site, multicompound SELDI-TOF-MS proteomics study in the context of a major European project focused on the use of 'omics technologies in pre-clinical toxicology. SELDI methodology has been previously applied in a number of studies with moderate success in the field of toxicoproteomics [16-19, 24, 25]. In general these have been discrete in nature, using either pattern matching-based methods to discriminate treatment groups [25], or identifying only a single protein as a putative toxicity related biomarker. A total of 11 proteins were identified from plasma and liver samples from drug treated animals. As suggested in a previous multi-site evaluation of the SELDI technology [15], we have demonstrated that with careful standardisation of protocols and materials, and suitable measurement of variance and biases, a robust dataset that is appropriate for statistical analysis across studies can be obtained.

A set of 11 proteins detected in plasma and/or liver samples by SELDI-TOF-MS has been correlated with the onset of toxicologic pathologies (liver hypertrophy, bile duct damage/necrosis, or nephrotoxicity) in treated rats and these proteins will be included for verification in further planned 
studies. The identified plasma proteins fall into the categories of acute phase/immune response (C reactive protein, haptoglobin, beta-2-microglobulin), or lipid transport (apolipoprotein Al, apolipoprotein All, apolipoprotein E, apolipoprotein M). Beta-2-microglobulin is a component of the major histocompatibility complex class I and is expressed in virtually all cell types. In the PredTox SELDI study, the level of beta-2-microglobulin was shown to be increased in the plasma of rats displaying all three pathologies under consideration (liver hypertrophy, bile duct damage/necrosis, and nephrotoxicity) and so by itself is unlikely to provide specificity but may be useful as a component of a biomarker panel. The candidate biomarkers identified in this study will most probably fall into this category given that the specificity for a given toxicologic pathology cannot be positively established at this time. As such, the candidates identified in this study should be included for evaluation in future studies in a blinded fashion to determine their predictive power.

The primary site of synthesis of apolipoproteins is in the liver (also to a limited extent in the gut), and changes in the levels of apolipoproteins in the liver and peripheral blood have been previously correlated with various types of liver damage in a number of studies [26-29]. Given the known origin of apolipoproteins, measurement of these markers could potentially add some level of specificity for liver toxicity, although a mechanistic link between increased apolipoproteins and the liver pathologies of interest is not apparent at this time. This probable lack of specificity underlines the need for further discovery work directed at sampling the proteome (both target organ tissue and plasma) to a greater depth in order to establish biomarkers with a higher level of specificity for a given toxicologic pathology.

One criterion on which differentially modulated peaks were selected for identification in this study was their presence at the same $\mathrm{m} / \mathrm{z}$ in spectra from both tissue and plasma samples, as it was hypothesised that these peaks could potentially represent the same protein. This, in fact, turned out to be the case for two proteins which were identified in liver and plasma samples. $\beta$-2-microglobulin and apolipoprotein All were both determined to be increased in the liver and plasma of treated 
animals displaying hypertrophy of the liver. This type of association, where increased concentrations of a secreted protein in an organ is reflected in an accessible biofluid such as the plasma, would represent an ideal situation for a biomarker candidate if specific linkage to the mechanism of toxicity could be established.

The other proteins determined to be up-regulated in liver were glutathione-s-transferase Yc1, a well described participant in the process of xenobiotic metabolism [30] and previously highlighted as a hepatotoxicity biomarker candidate [31, 32], and phosphatidylethanolamine binding protein (a.k.a. raf kinase inhibitor protein), for which modulation has been previously associated with liver cirrhosis/hepatocellular carcinoma [33], and hepatomegaly [34].

The chief drawback of the SELDI method, as practised in this project, is the bottleneck in identifying proteins after differential profiling and statistical analysis (although this identification step need only be done once). This feature of the approach has constrained our ability to attempt an effective analysis across 'omics datasets in the PredTox project. In particular, a comparison with transcriptomics data may have been fruitful if more features in the SELDI dataset had been assigned protein annotations. A second issue is the depth to which the proteome was sampled by this method. Depletion of abundant proteins from plasma samples certainly aided in the number of detectable features; however, the depletion of albumin from the sample is undoubtedly removing a substantial number of low molecular weight albumin-bound proteins that could be potentially important biomarker candidates [35]. An attempt was made in the pilot studies to elute these fractions from the depletion columns using instructions from the manufacturer; however, the recovery was found to be highly variable, and so these fractions were not analysed in the main studies. Further fractionation/enrichment strategies would be helpful in identifying biomarker candidates which are more specific for a given toxicologic pathology. This is true for both plasma and tissue studies. One such technology which has general sample applicability is combinatorial peptide ligand libraries (commercialised as ProteoMiner by Bio-Rad Laboratories) [36]. An alternative 
approach that could address some of these shortcomings is to introduce a technology such as labelfree LC-MS which could provide a greater depth of quantitative information with greatly increased numbers of protein annotations for profiled features [37]. A second solution may be to adopt a targeted strategy whereby candidate markers from transcriptomic and proteomics studies are specifically assayed by LC-MS based strategies, such as multiple reaction monitoring [38]. These are attractive prospects but it should be noted that the throughput of sample analysis would be decreased and the prospect of profiling the required number of samples may become untenable, so it seems that with current proteomic technologies a compromise between power and throughput must still be reached. In addition, the continuing development of MALDI-TOF profiling approaches incorporating sample preparation such as solid phase extraction which might overcome some of the limitations of SELDI-TOF as described in this study is ongoing, and may provide a more feasible alternative for high-throughput pre-clinical/clinical proteomics studies in the near future [39-41]. The ongoing improvement of mass spectrometry-based proteomics technologies to enable greater depth of coverage and throughput should facilitate mechanism specific toxicity biomarkers to emerge [10].

These issues, and others, are currently under consideration to determine how best to move forward in the field of predictive toxicology with respect to molecular profiling technologies. One possibility would be a follow-on project to PredTox which would aim to build on the current dataset with many more additional compounds, and an 'omics data collection strategy that would be refined based on the unique experience of the PredTox project. Expansion of the knowledgebase with additional well characterised compounds would provide the opportunity to further increase and refine the mechanistic knowledge associated with specific toxicologic pathologies and define biomarkers associated with their prediction and monitoring. 


\section{Acknowledgements}

We would like, in particular, to thank all other members of the InnoMed PredTox Consortium.

Funding of the Integrated Project, InnoMed, is acknowledged under the European Union's Sixth

Framework Programme. The UCD Conway Institute and the Proteome Research Centre is funded by

the Programme for Research in Third Level Institutions (PRTLI), as administered by the Higher

Education Authority (HEA) of Ireland. Equipment funding is acknowledged from the Health Research

Board of Ireland, Science Foundation Ireland and University College Dublin.

Conflict of interest statement

No conflicts of interest are declared.

\section{References}

[1] Kola, I., Landis, J., Can the pharmaceutical industry reduce attrition rates? Nature reviews 2004, 3, 711-715.

[2] Kramer, J. A., Sagartz, J. E., Morris, D. L., The application of discovery toxicology and pathology towards the design of safer pharmaceutical lead candidates. Nature reviews 2007, 6, 636-649.

[3] DiMasi, J. A., Hansen, R. W., Grabowski, H. G., The price of innovation: new estimates of drug development costs. J Health Econ 2003, 22, 151-185.

[4] Waters, M. D., Fostel, J. M., Toxicogenomics and systems toxicology: aims and prospects. Nat Rev Genet 2004, 5, 936-948.

[5] Heijne, W. H., Kienhuis, A. S., van Ommen, B., Stierum, R. H., Groten, J. P., Systems toxicology: applications of toxicogenomics, transcriptomics, proteomics and metabolomics in toxicology. Expert Rev Proteomics 2005, 2, 767-780.

[6] Suter, L., Babiss, L. E., Wheeldon, E. B., Toxicogenomics in predictive toxicology in drug development. Chem Biol 2004, 11, 161-171.

[7] Maggioli, J., Hoover, A., Weng, L., Toxicogenomic analysis methods for predictive toxicology. J Pharmacol Toxicol Methods 2005, 53, 31-37.

[8] Merrick, B. A., The plasma proteome, adductome and idiosyncratic toxicity in toxicoproteomics research. Briefings in functional genomics \& proteomics 2008.

[9] Wetmore, B. A., Merrick, B. A., Toxicoproteomics: proteomics applied to toxicology and pathology. Toxicologic pathology 2004, 32, 619-642. 
[10] Collins, B. C., Clarke, A., Kitteringham, N. R., Gallagher, W. M., Pennington, S. R., Use of proteomics for the discovery of early markers of drug toxicity. Expert Opin Drug Metab Toxicol 2007, 3, 689-704.

[11] Craig, A., Sidaway, J., Holmes, E., Orton, T., et al., Systems toxicology: Integrated genomic, proteomic and metabonomic analysis of methapyrilene induced hepatotoxicity in the rat. Journal of proteome research 2006, 5, 1586-1601.

[12] Gallagher, W. M., Tweats, D., Koenig, J., Omic profiling for drug safety assessment: current trends and public-private partnerships. Drug discovery today 2009, 14, 337-342.

[13] Mulrane, L., Rexhepaj, E., Smart, V., Callanan, J. J., et al., Creation of a digital slide and tissue microarray resource from a multi-institutional predictive toxicology study in the rat: an initial report from the PredTox group. Exp Toxicol Pathol 2008, 60, 235-245.

[14] Tang, N., Tornatore, P., Weinberger, S. R., Current developments in SELDI affinity technology. Mass spectrometry reviews 2004, 23, 34-44.

[15] Rai, A. J., Stemmer, P. M., Zhang, Z., Adam, B. L., et al., Analysis of Human Proteome Organization Plasma Proteome Project (HUPO PPP) reference specimens using surface enhanced laser desorption/ionization-time of flight (SELDI-TOF) mass spectrometry: multi-institution correlation of spectra and identification of biomarkers. Proteomics 2005, 5, 3467-3474.

[16] Beyer, S., Walter, Y., Hellmann, J., Kramer, P. J., et al., Comparison of software tools to improve the detection of carcinogen induced changes in the rat liver proteome by analyzing SELDITOF-MS spectra. Journal of proteome research 2006, 5, 254-261.

[17] Bjornstad, A., Larsen, B. K., Skadsheim, A., Jones, M. B., Andersen, O. K., The potential of ecotoxicoproteomics in environmental monitoring: biomarker profiling in mussel plasma using ProteinChip array technology. Journal of toxicology and environmental health 2006, 69, 77-96. [18] Dare, T. O., Davies, H. A., Turton, J. A., Lomas, L., et al., Application of surface-enhanced laser desorption/ionization technology to the detection and identification of urinary parvalbuminalpha: a biomarker of compound-induced skeletal muscle toxicity in the rat. Electrophoresis 2002, 23, 3241-3251.

[19] Hogstrand, C., Balesaria, S., Glover, C. N., Application of genomics and proteomics for study of the integrated response to zinc exposure in a non-model fish species, the rainbow trout. Comp Biochem Physiol B Biochem Mol Biol 2002, 133, 523-535.

[20] Bradford, M. M., A rapid and sensitive method for the quantitation of microgram quantities of protein utilizing the principle of protein-dye binding. Analytical biochemistry 1976, 72, 248-254.

[21] Fella, K., Gluckmann, M., Hellmann, J., Karas, M., et al., Use of two-dimensional gel electrophoresis in predictive toxicology: identification of potential early protein biomarkers in chemically induced hepatocarcinogenesis. Proteomics 2005, 5, 1914-1927.

[22] Candiano, G., Bruschi, M., Musante, L., Santucci, L., et al., Blue silver: a very sensitive colloidal Coomassie G-250 staining for proteome analysis. Electrophoresis 2004, 25, 1327-1333.

[23] Anderson, N. L., Anderson, N. G., The human plasma proteome: history, character, and diagnostic prospects. Mol Cell Proteomics 2002, 1, 845-867.

[24] Zhu, Z., Edwards, R. J., Boobis, A. R., Proteomic analysis of human breast cell lines using SELDITOF MS shows that mixtures of estrogenic compounds exhibit simple similar action (concentration additivity). Toxicology letters 2008, 181, 93-103.

[25] Petricoin, E. F., Rajapaske, V., Herman, E. H., Arekani, A. M., et al., Toxicoproteomics: serum proteomic pattern diagnostics for early detection of drug induced cardiac toxicities and cardioprotection. Toxicologic pathology 2004, 32 Suppl 1, 122-130.

[26] Boll, M., Weber, L. W., Becker, E., Stampfl, A., Hepatocyte damage induced by carbon tetrachloride: inhibited lipoprotein secretion and changed lipoprotein composition. Zeitschrift fur Naturforschung 2001, 56, 283-290.

[27] Chao, Y. S., Pickett, C. B., Yamin, T. T., Guo, L. S., et al., Phenobarbital induces rat liver apolipoprotein A-I mRNA. Molecular pharmacology 1985, 27, 394-398. 
[28] Kleno, T. G., Leonardsen, L. R., Kjeldal, H. O., Laursen, S. M., et al., Mechanisms of hydrazine toxicity in rat liver investigated by proteomics and multivariate data analysis. Proteomics 2004, 4, 868-880.

[29] Amacher, D. E., Adler, R., Herath, A., Townsend, R. R., Use of proteomic methods to identify serum biomarkers associated with rat liver toxicity or hypertrophy. Clinical chemistry 2005, 51, 1796-1803.

[30] Salinas, A. E., Wong, M. G., Glutathione S-transferases--a review. Current medicinal chemistry 1999, 6, 279-309.

[31] Shaw, M., The use of histologically defined specific biomarkers in drug development with special reference to the glutathione S-transferases. Cancer Biomark 2005, 1, 69-74.

[32] Ozer, J., Ratner, M., Shaw, M., Bailey, W., Schomaker, S., The current state of serum biomarkers of hepatotoxicity. Toxicology 2008, 245, 194-205.

[33] Schuierer, M. M., Bataille, F., Weiss, T. S., Hellerbrand, C., Bosserhoff, A. K., Raf kinase inhibitor protein is downregulated in hepatocellular carcinoma. Oncology reports 2006, 16, 451456.

[34] Nilsen, A. J., Landin, M. A., Haug, K. H., Fonnum, F., et al., Comparative hepatic gene expression profiling of rats treated with $1 \mathrm{H}, 1 \mathrm{H}, 2 \mathrm{H}, 2 \mathrm{H}$-heptadecafluorodecan-1-ol or with pentadecafluorooctanoic acid. Physiological genomics 2008, 34, 285-303.

[35] Lopez, M. F., Mikulskis, A., Kuzdzal, S., Golenko, E., et al., A novel, high-throughput workflow for discovery and identification of serum carrier protein-bound peptide biomarker candidates in ovarian cancer samples. Clinical chemistry 2007, 53, 1067-1074.

[36] Boschetti, E., Righetti, P. G., The ProteoMiner in the proteomic arena: a non-depleting tool for discovering low-abundance species. Journal of proteomics 2008, 71, 255-264.

[37] America, A. H., Cordewener, J. H., Comparative LC-MS: a landscape of peaks and valleys. Proteomics 2008, 8, 731-749.

[38] Kuster, B., Schirle, M., Mallick, P., Aebersold, R., Scoring proteomes with proteotypic peptide probes. Nature reviews 2005, 6, 577-583.

[39] Tiss, A., Smith, C., Camuzeaux, S., Kabir, M., et al., Serum peptide profiling using MALDI mass spectrometry: avoiding the pitfalls of coated magnetic beads using well-established ZipTip technology. Proteomics 2007, 7 Suppl 1, 77-89.

[40] Callesen, A. K., Madsen, J. S., Vach, W., Kruse, T. A., et al., Serum protein profiling by solid phase extraction and mass spectrometry: a future diagnostics tool? Proteomics 2009, 9, 14281441.

[41] Albrethsen, J., Reproducibility in protein profiling by MALDI-TOF mass spectrometry. Clinical chemistry 2007, 53, 852-858.

Figure Legends

Figure 1 - Effect of depletion of albumin and IgG from plasma on SELDI spectra

Albumin and IgG were affinity depleted from plasma samples in order to increase the number of lower abundance features detected. A significant reduction in the intensity of proposed albumin 
peaks (singly charged, doubly charged, and singly charged dimer), as well as the proposed IgG peak, are clearly visible.

Figure 2 - Within study reproducibility of SELDI profiling of reference sample

The within study variation, as measured by \% coefficient of variation in the reference plasma sample, is plotted with respect to the array chemistry, sample type, and site of data collection. The overall mean \% coefficient of variation was calculated to be $23 \%$.

Figure 3 - Variation of reference sample spectra visualised by principal components analysis

Reference sample spectra collected from every array measured throughout the project were subjected to principal components analysis. The upper panel is annotated by site of data collection and the lower panel is annotated by individual compound study. It is clear that the variance from site to site is greater than that for individual compound studies where data was collected from a single site.

Figure 4 - Representative reference sample spectra on the Q10 pH9 surface from all 3 sites of SELDI data collection

A visual inspection of the reference sample spectra from all 3 participating sites shows that virtually all peaks are represented at each site but with differing intensities. Panel (a) shows the spectra with the intensity axis scaled to the highest peak. Panel (b) shows the same spectra with the intensity axis scaled to show lower abundance peaks. 
Figure 5 - Summary of significantly regulated peaks across all compound studies

The number of peaks differentially regulated at two statistical significance cut-offs in each compound study are plotted with respect to the array surface used and the sample type (depleted plasma and target organ, i.e. liver or kidney). The cut-offs used were change greater than $50 \%$ and Mann-Whitney $p$ value less than 0.05 , or fold change greater than 2 and Mann-Whitney $p$ value less than 0.01 (for some studies in which animals were excluded due to issues in the in vivo study or for spectral quality control, the second $p$ value cut-off was raised to 0.015 as a value lower than 0.01 was not possible in a statistical test using ranked data such as Mann-Whitney). Study FP016LY was excluded from the analysis due to irregularities in the in vivo experiment.

Figure 6 - Intensity of the 11,645 Da peak ( $\beta$-2-microglobulin) from plasma samples in 3 compound studies classified in the liver bile duct damage/necrosis histopathology group

The intensity of the 11,645 Da peak (subsequently identified as $\beta$-2-microglobulin) was consistently and significantly (see Table 1) increased in the blood plasma of animals in the bile duct damage/necrosis histopathology group who received the high dose treatment at both day 4 and day 15. The intensity of the vehicle control groups for the compound studies FP005ME and FP014SC are at a comparable level, as the SELDI data for both of these studies were collected at a single site (UCD); however, the SELDI data for the compound study FP007SE was collected at a different site (Merck-Serono).

\section{Figure 7 - Identification of $\mathbf{1 1 . 6}$ kDa peak as beta-2-microglobulin from depleted plasma}

The identification workflow for beta-2-microglobulin is outlined. Similar workflows, using different combinations of anion exchange and reversed phase chromatography, isoelectric focusing, and/or 
SDS-PAGE were employed for the identification of all peaks of interest. Panel (a) shows a selection of fractions from preparative isoelectric focussing of depleted plasma monitored by SELDI-TOF-MS in which the $11.6 \mathrm{kDa}$ peak is focussing in fraction 17. This fractionation step was carried out in parallel using pools of samples with high and low levels of the target protein (day 15 vehicle treated group versus day 15 high dose treated group). The increased peak height is maintained between the vehicle treated and high dose treated groups. In general, either preparative isoelectric focusing or strong anion exchange chromatography was used as the initial fractionation technique for identification efforts. Panel (b) shows a further purification of the peak from the preparative isoelectric focusing of interest by fractional elution from a reversed phase resin. The reversed phase separation step also acts as a cleanup step for polar or ionic solutes such that the sample can be concentrated by evaporation prior to 1-dimensional electrophoresis. Panel (c) shows the proteins in the reversed phase fractions separated by 1-dimensional electrophoresis. The protein band highlighted in the $35 \%$ ACN lane was thought to represent the $11.6 \mathrm{kDa}$ peak based on the mobility and intensity and so a gel plug was punched and subjected to passive elution so that the protein mass could be confirmed by SELDI-TOF-MS (shown in panel D). The increased mass and broadening of the peak with respect to the previous purification steps is consistent with multiple oxidation events due to sample preparation for 1-dimensional electrophoresis (boiling, etc. - acrylamide modification and formylation are also frequently observed). This is commonly observed and is consistent with the MS/MS spectra from the tryptic digestion of this protein, which showed multiple oxidised peptides. An example of a peptide fragmentation spectrum (peptide TPQIQVTSR) is shown in panel (e). Panel (f) shows the significant hits from the Mascot search engine with the beta-2microglobulin annotation highlighted (probability based Mowse score $=1993)$. There are a number of hits in the Mowse score range 100-800, but these all represent hits to known contaminant proteins (trypsin, keratins, etc.) which were included in the search database. Panel (g) shows the 
Mascot output including peptides identified for beta-2-microglobulin demonstrating very high confidence in the annotation. 
Tables

Table 1 - Fold change and p-values for the 11,645 Da peak ( $\beta$-2-microglobulin) from plasma samples in 3 compound studies classified in the liver bile duct damage/necrosis histopathology group (data corresponding to Figure 5)

\begin{tabular}{|l|l|l|l|l|}
\hline Study & Dose & Time point & $\begin{array}{l}\text { Fold change vs. } \\
\text { vehicle control } \\
\text { (median) }\end{array}$ & $\begin{array}{l}\text { p-value } \\
\text { (Mann-Whitney) }\end{array}$ \\
\hline \multirow{3}{*}{ FP005ME } & \multirow{2}{*}{ Low Dose } & Day 4 & 1.19 & 0.251 \\
\cline { 3 - 5 } & & Day 15 & 1.42 & 0.009 \\
\cline { 3 - 5 } & \multirow{2}{*}{ High Dose } & Day 4 & 2.61 & 0.009 \\
\cline { 3 - 5 } & Day 15 & 2.01 & 0.009 \\
\hline \multirow{3}{*}{ FP07SE } & \multirow{2}{*}{ Low Dose } & Day 4 & 1.11 & 0.251 \\
\cline { 3 - 5 } & & Day 15 & 0.98 & 0.917 \\
\cline { 3 - 5 } & \multirow{2}{*}{ High Dose } & Day 4 & 1.36 & 0.028 \\
\cline { 3 - 5 } & & Day 15 & 1.55 & 0.014 \\
\hline & \multirow{2}{*}{ Low Dose } & Day 4 & 0.70 & 0.602 \\
\cline { 3 - 5 } & & Day 15 & 0.79 & 0.462 \\
\cline { 3 - 5 } & \multirow{2}{*}{ High Dose } & Day 4 & 2.20 & 0.117 \\
\cline { 3 - 5 } & & Day 15 & 2.16 & \\
\hline
\end{tabular}


Table 2 - Summary of identified plasma proteins modulated on drug treatment

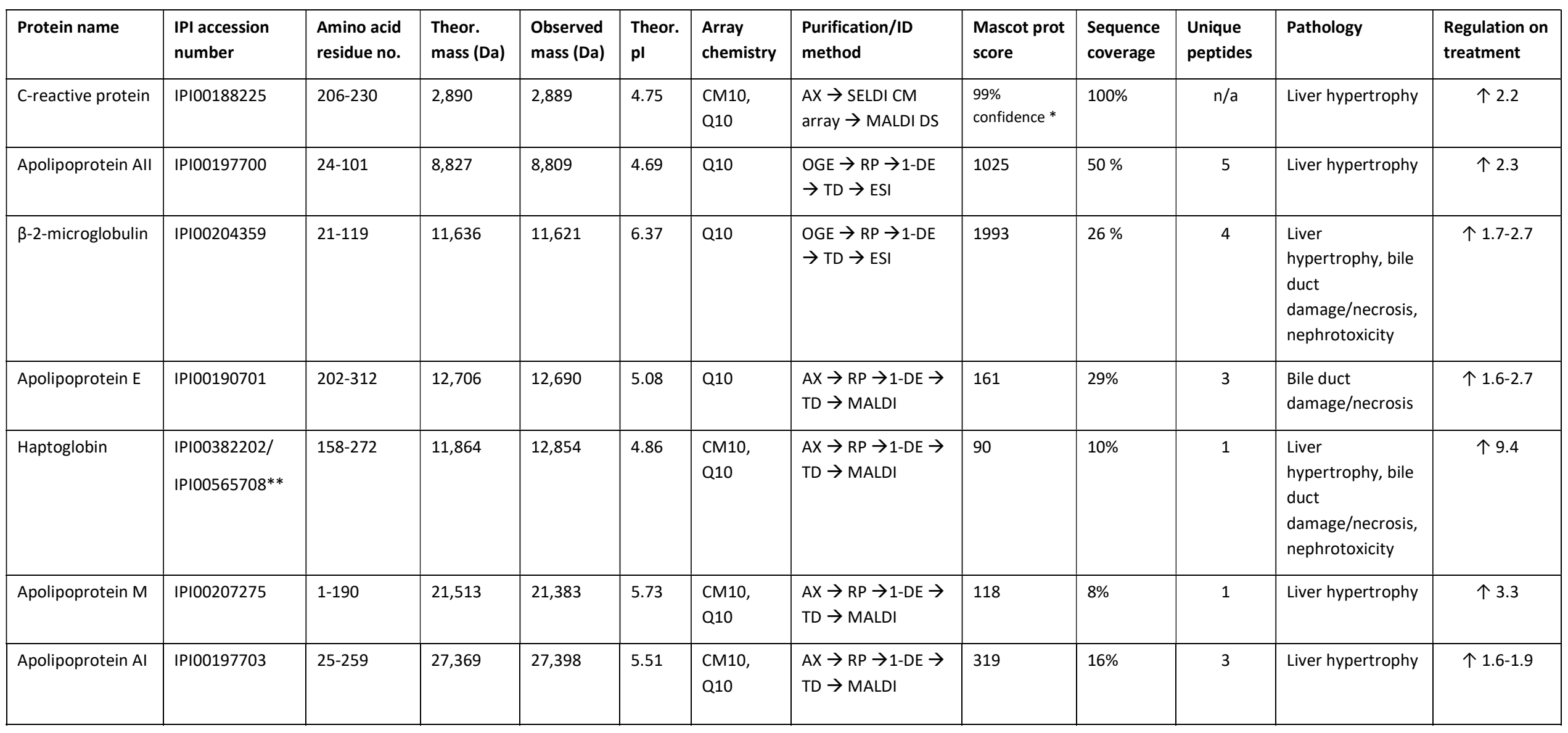

See footnote for Table 3 for abbreviation definitions 
Table 3 - Summary of identified liver proteins modulated on drug treatment

\begin{tabular}{|c|c|c|c|c|c|c|c|c|c|c|c|c|}
\hline Protein name & $\begin{array}{l}\text { IPI accession } \\
\text { number }\end{array}$ & $\begin{array}{l}\text { Amino acid } \\
\text { residue no. }\end{array}$ & $\begin{array}{l}\text { Theor. } \\
\text { mass (Da) }\end{array}$ & $\begin{array}{l}\text { Observed } \\
\text { mass (Da) }\end{array}$ & $\begin{array}{l}\text { Theor. } \\
\text { pl }\end{array}$ & $\begin{array}{l}\text { Array } \\
\text { chemistry }\end{array}$ & $\begin{array}{l}\text { Purification/ID } \\
\text { method }\end{array}$ & $\begin{array}{l}\text { Mascot } \\
\text { prot score }\end{array}$ & $\begin{array}{l}\text { Sequence } \\
\text { coverage }\end{array}$ & $\begin{array}{l}\text { Unique } \\
\text { peptides }\end{array}$ & Pathology & $\begin{array}{l}\text { Regulation on } \\
\text { treatment }\end{array}$ \\
\hline Apolipoprotein All & IPI00197700 & 24-101 & 8,827 & 8,806 & 4.69 & Q10 & $\begin{array}{l}\mathrm{AX} \rightarrow \mathrm{RP} \rightarrow 1-\mathrm{DE} \\
\rightarrow \mathrm{TD} \rightarrow \mathrm{MALDI}\end{array}$ & 125 & $21 \%$ & 2 & Liver hypertrophy & $\uparrow 2.2$ \\
\hline$\beta$-2-microglobulin & IPI00204359 & 21-119 & 11,636 & 11,645 & 6.37 & $\begin{array}{l}\text { CM10, } \\
\text { Q10 }\end{array}$ & $\begin{array}{l}\mathrm{AX} \rightarrow \mathrm{RP} \rightarrow 1-\mathrm{DE} \\
\rightarrow \mathrm{TD} \rightarrow \mathrm{MALDI}\end{array}$ & 35 & $9 \%$ & 1 & $\begin{array}{l}\text { Liver hypertrophy, } \\
\text { bile duct } \\
\text { damage/necrosis }\end{array}$ & $\uparrow 1.5$ \\
\hline $\begin{array}{l}\text { Phosphatidyletha } \\
\text { nolamine binding } \\
\text { protein }\end{array}$ & IPI00230937 & $2-187$ & 20,670 & 20,700 & 5.48 & $\begin{array}{l}\text { CM10, } \\
\text { Q10 }\end{array}$ & $\begin{array}{l}\mathrm{AX} \rightarrow \mathrm{RP} \rightarrow 1-\mathrm{DE} \\
\rightarrow \mathrm{TD} \rightarrow \mathrm{MALDI}\end{array}$ & 147 & $32 \%$ & 3 & $\begin{array}{l}\text { bile duct } \\
\text { damage/necrosis }\end{array}$ & 个 1.5-1.9 \\
\hline $\begin{array}{l}\text { Glutathione-S- } \\
\text { transferase Yc1 }\end{array}$ & IPI00231150 & $2-221$ & 25,188 & 25,193 & 8.83 & CM10 & $\begin{array}{l}\mathrm{AX} \rightarrow \mathrm{TCA} \rightarrow \text { 1-DE } \\
\rightarrow \mathrm{TD} \rightarrow \mathrm{MALDI}\end{array}$ & 434 & $28 \%$ & 3 & $\begin{array}{l}\text { bile duct } \\
\text { damage/necrosis }\end{array}$ & 个 1.5-1.8 \\
\hline
\end{tabular}

OGE - off gel electrophoresis, SAX - strong anion exchange chromatography, RP - reversed phase chromatography, 1-DE - 1 dimensional electrophoresis,

TD - tryptic digest, MALDI - MALDI-TOF/TOF tandem mass spectrometry, ESI - electrospray linear ion trap tandem mass spectrometry, DS - direct

sequencing by MALDI-TOF/TOF. Sequence coverage, theoretical mass, and theoretical pl are calculated based on the proposed fragment amino acid sequence, and not on the full-length protein sequence from the IPI database. Sequence coverage is calculated from peptides which have high confidence MS/MS spectral assignments, and do not include peptides where only the precursor mass was observed. * indicates a Protein Pilot confidence score. ** indicates that the protein fragment measured (residue 158-272) maps to isoform-1 (IPI00565708) and isoform-2 (IPI00382202) of haptoglobin. 
Table 4 - Fold change of beta-2-microglobulin versus time matched vehicle controls as measured by SELDI peak intensity and ELISA in study FP014SC liver protein extracts.

\begin{tabular}{|l|c|c|c|c|}
\hline \multirow{2}{*}{ Group } & \multicolumn{2}{|c|}{ SELDI } & \multicolumn{2}{c|}{ ELISA } \\
\cline { 2 - 5 } & $\begin{array}{c}\text { Median } \\
\text { fold change }\end{array}$ & $\begin{array}{c}\text { Mann-Whitney } \\
\mathbf{p} \text {-value }\end{array}$ & $\begin{array}{c}\text { Median } \\
\text { fold change }\end{array}$ & $\begin{array}{c}\text { Mann-Whitney } \\
\mathbf{p} \text {-value }\end{array}$ \\
\hline Day 4 - low/vehicle & 1.08 & 0.465 & 0.85 & 0.754 \\
\hline Day 4 - high/vehicle & 0.93 & 0.602 & 0.92 & 0.602 \\
\hline Day 15 - low/vehicle & 1.14 & 0.465 & 1.22 & 0.142 \\
\hline Day 15 - high/vehicle & $\mathbf{1 . 4 9}$ & $\mathbf{0 . 0 0 9}$ & $\mathbf{1 . 4 8}$ & $\mathbf{0 . 0 1 4}$ \\
\hline
\end{tabular}




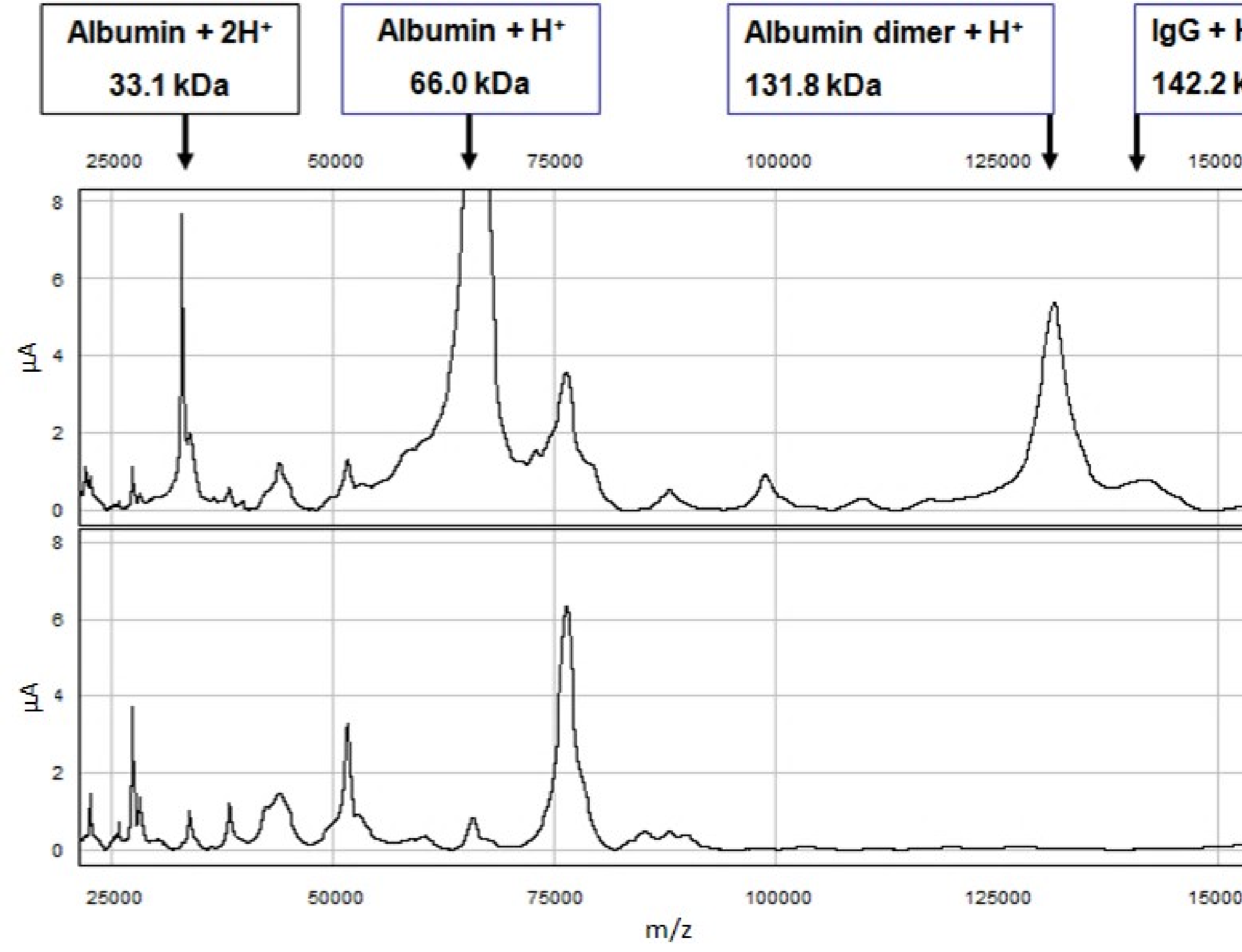




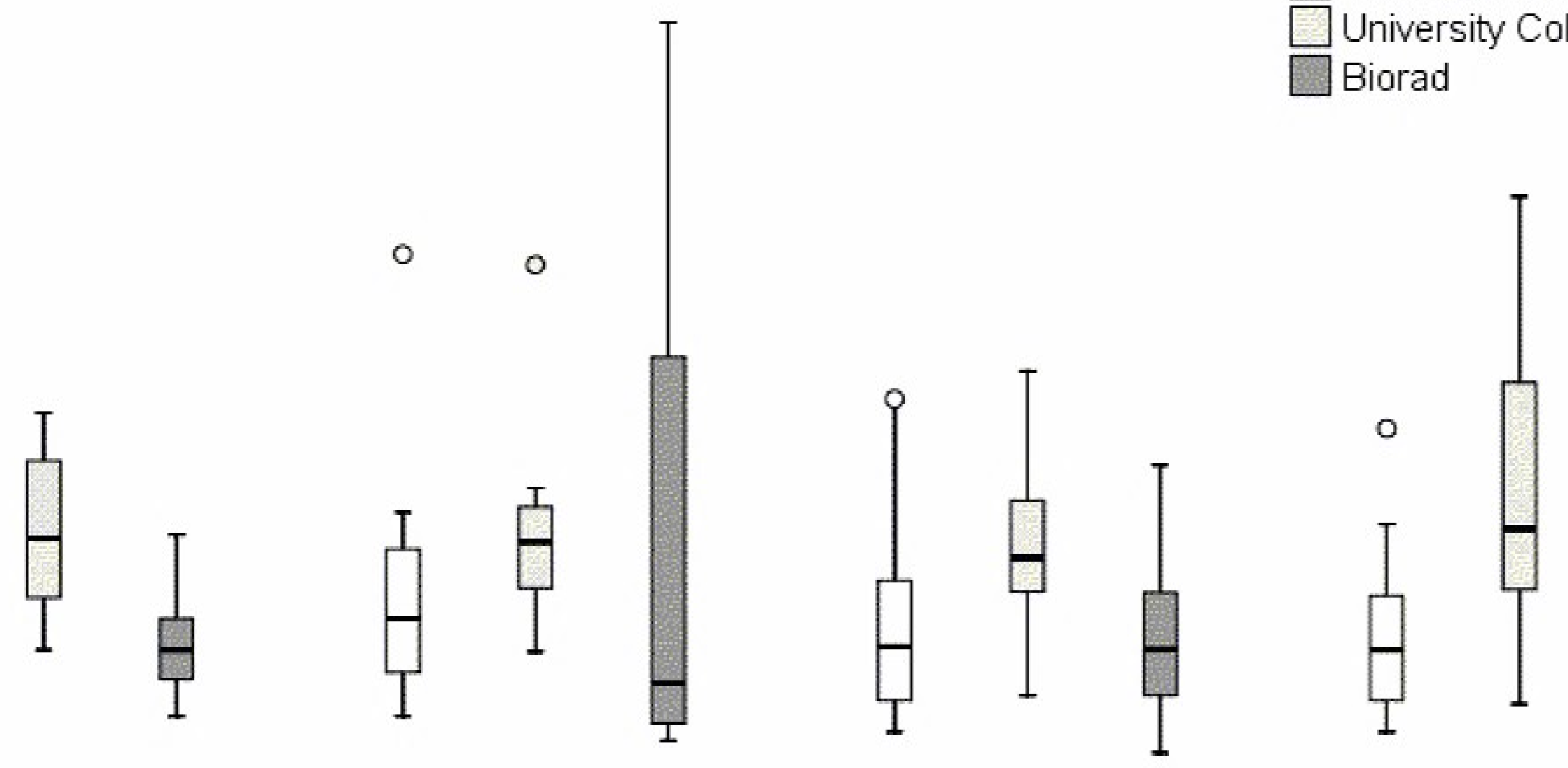




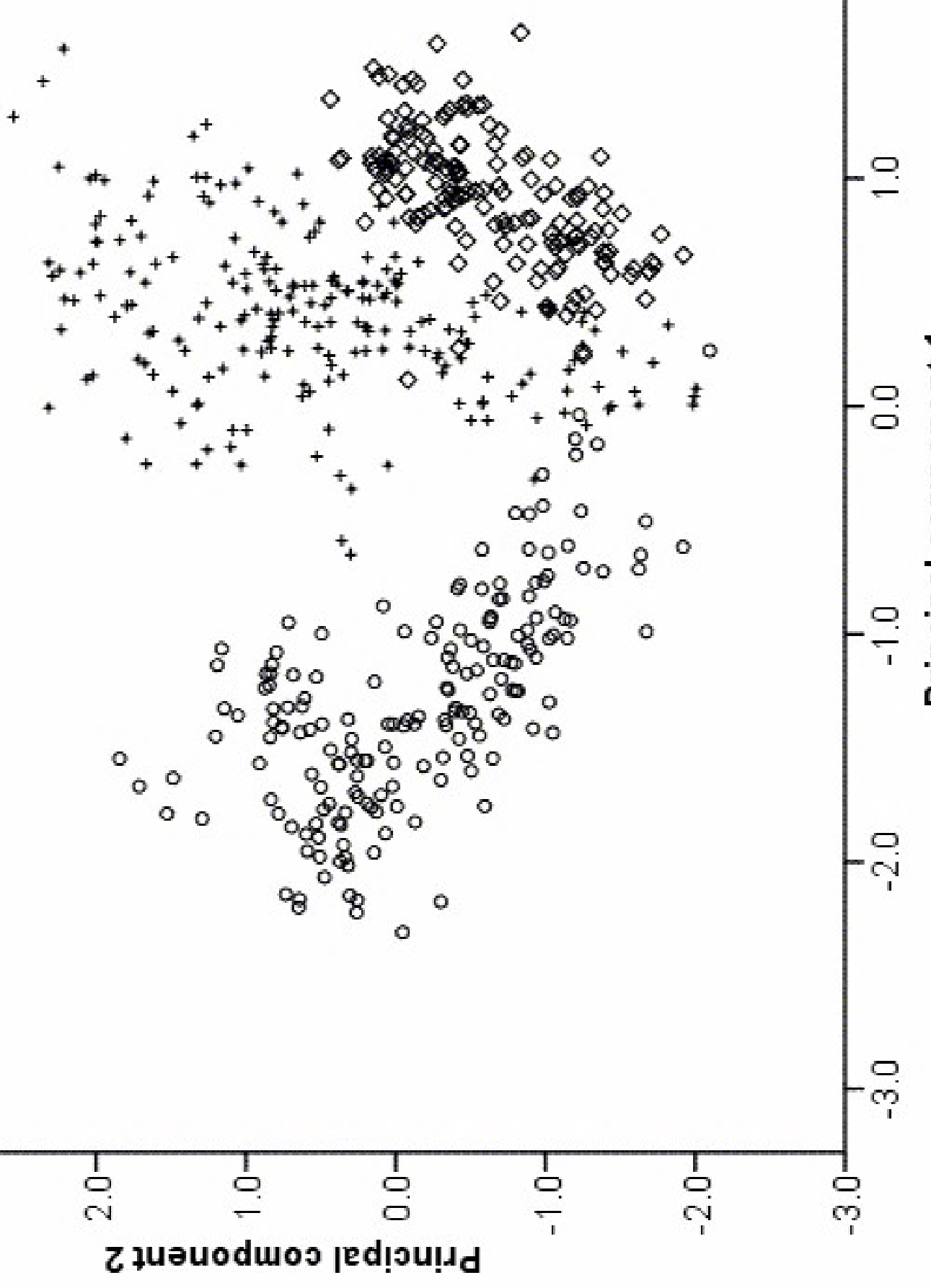

$a$

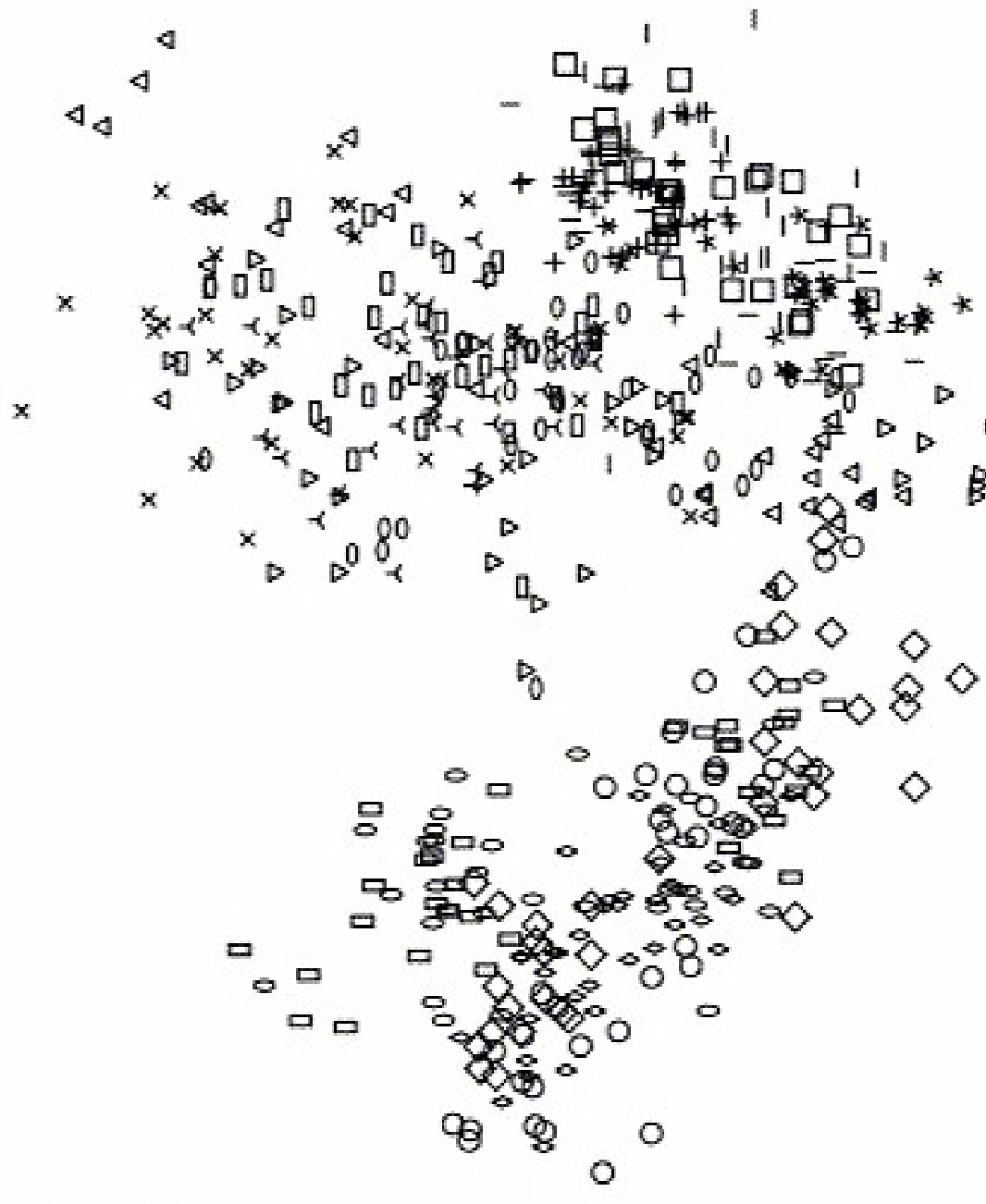

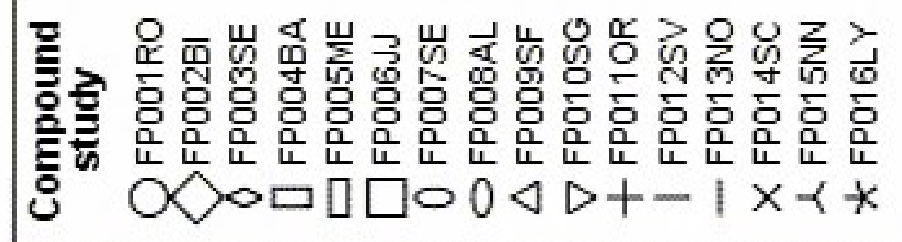

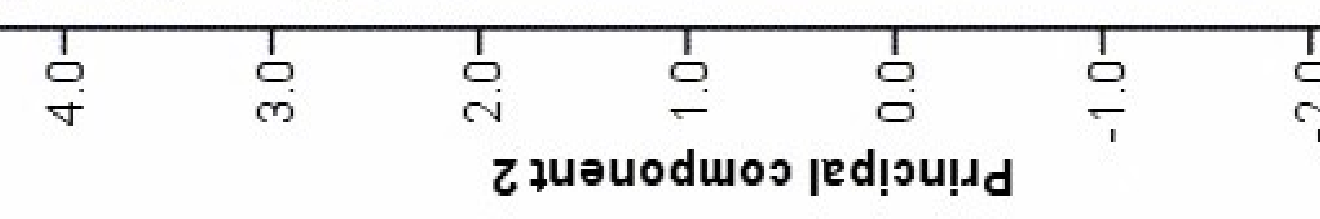




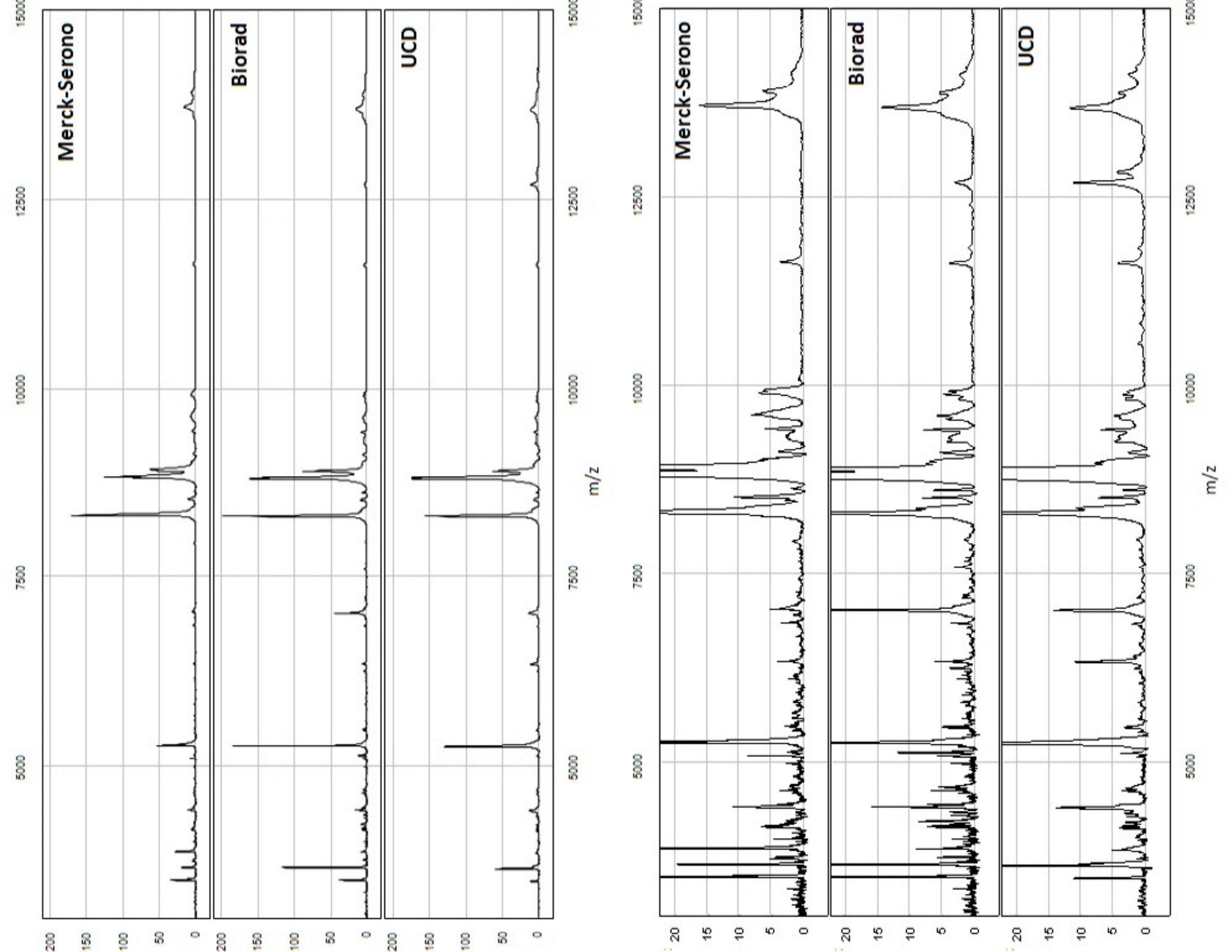




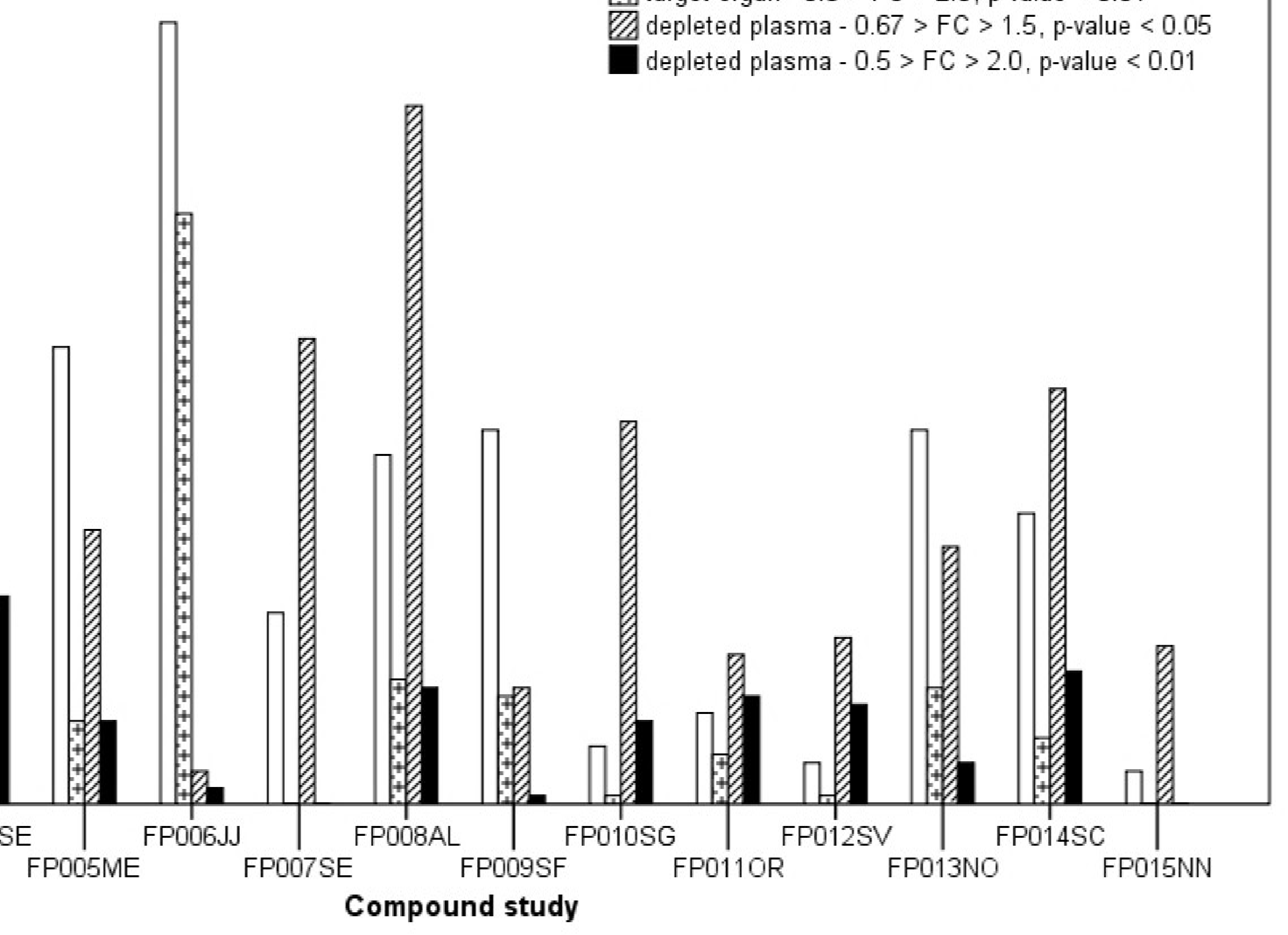




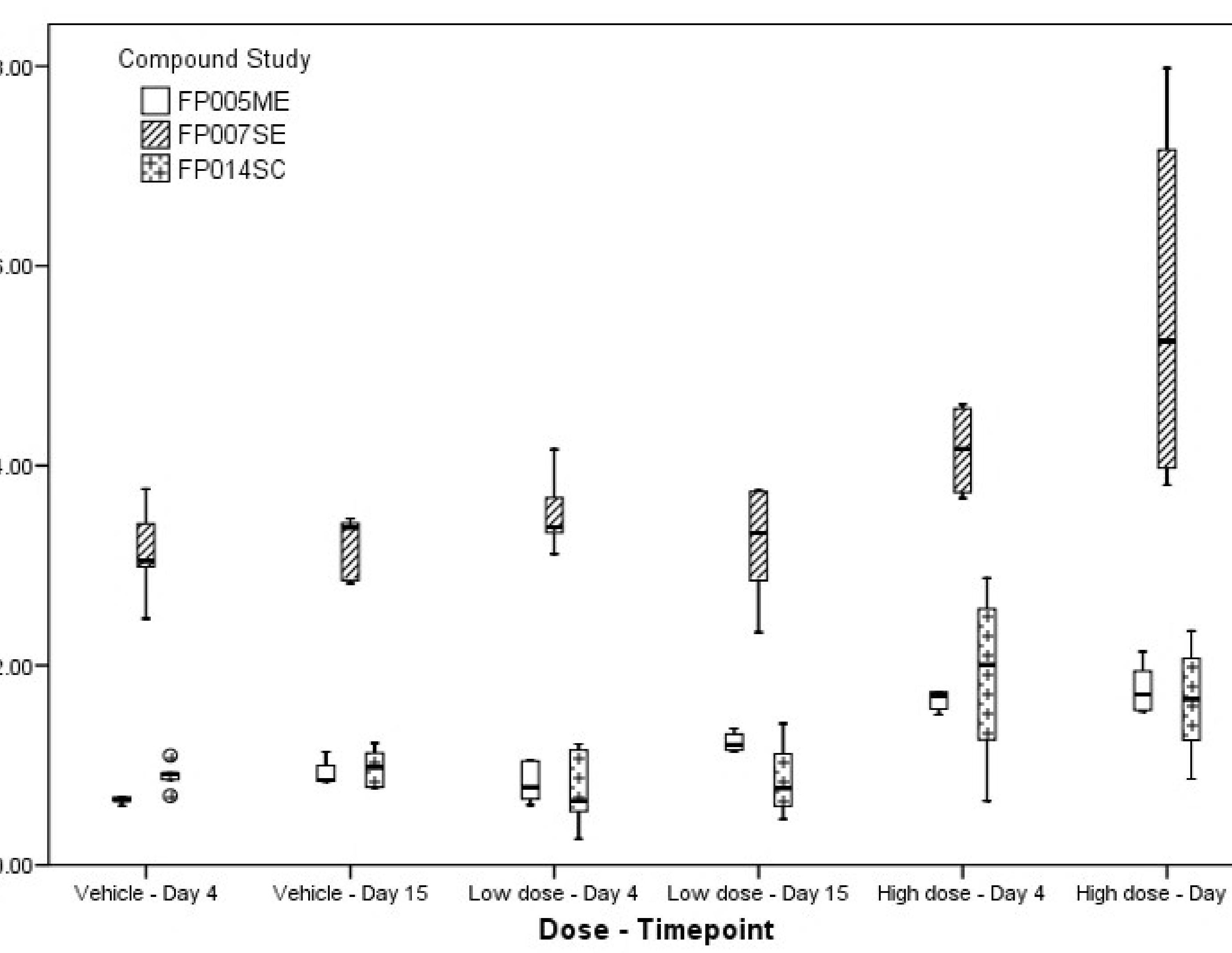




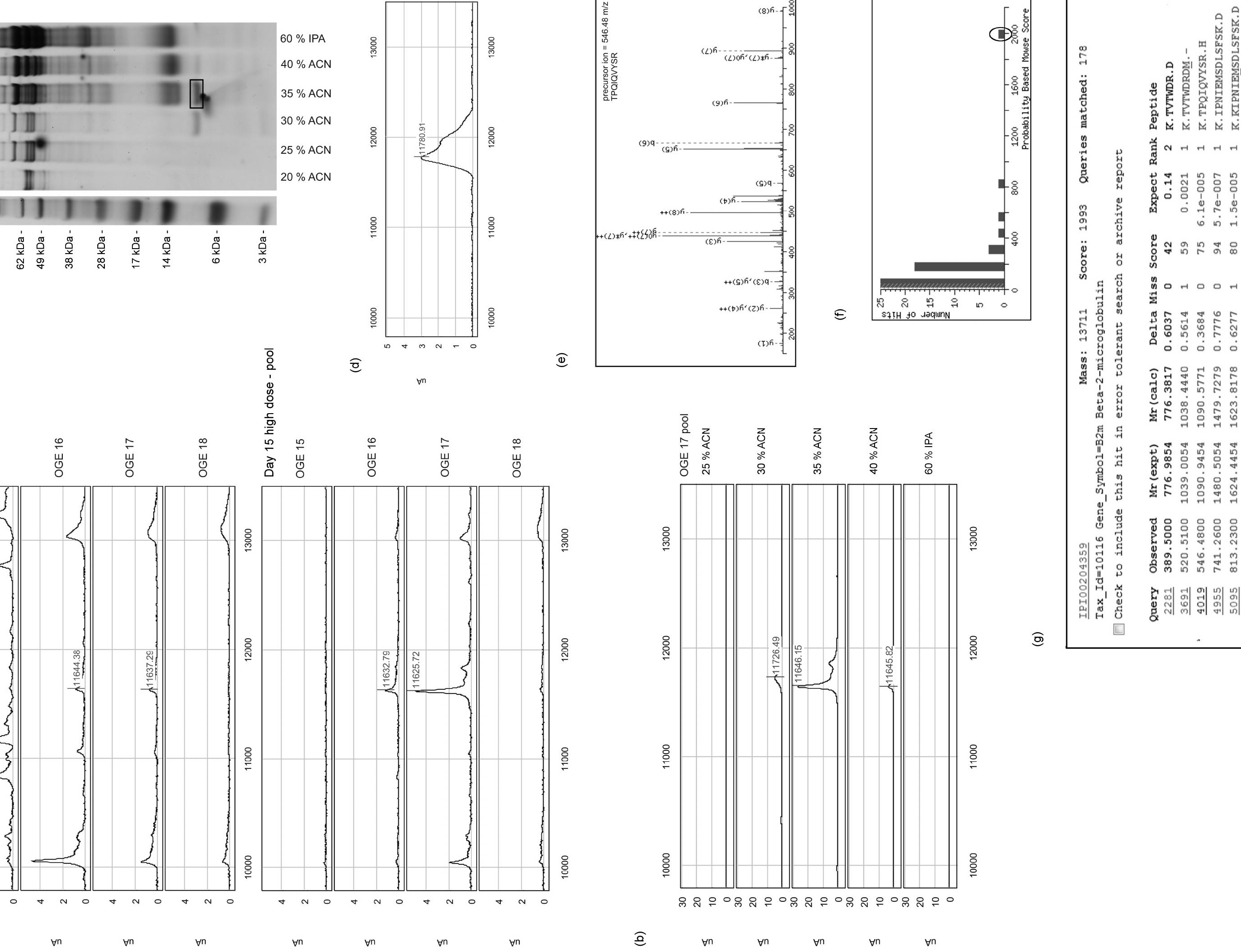

